\title{
A Hybrid Global Optimization Algorithm Based on Wind Driven Optimization and Differential Evolution
}

\author{
Zongfan Bao, ${ }^{1}$ Yongquan Zhou, ${ }^{1,2}$ Liangliang $\mathrm{Li}^{1}{ }^{1}$ and Mingzhi Ma ${ }^{1}$ \\ ${ }^{1}$ College of Information Science and Engineering, Guangxi University for Nationalities, Nanning, Guangxi 530006, China \\ ${ }^{2}$ Key Laboratory of Guangxi High School Complex System and Computational Intelligence, Nanning 530006, China \\ Correspondence should be addressed to Yongquan Zhou; yongquanzhou@126.com
}

Received 7 June 2015; Accepted 22 July 2015

Academic Editor: Alessandro Salvini

Copyright (c) 2015 Zongfan Bao et al. This is an open access article distributed under the Creative Commons Attribution License, which permits unrestricted use, distribution, and reproduction in any medium, provided the original work is properly cited.

\begin{abstract}
This paper presents a new hybrid global optimization algorithm, which is based on the wind driven optimization (WDO) and differential evolution (DE), named WDO-DE algorithm. The WDO-DE algorithm is based on a double population evolution strategy, the individuals in a population evolved by wind driven optimization algorithm, and a population of individuals evolved from difference operation. The populations of individuals both in WDO and DE employ an information sharing mechanism to implement coevolution. This paper chose fifteen benchmark functions to have a test. The experimental results show that the proposed algorithm can be feasible in both low-dimensional and high-dimensional cases. Compared to GA-PSO, WDO, DE, PSO, and BA algorithm, the convergence speed and precision of WDO-DE are higher. This hybridization showed a better optimization performance and robustness and significantly improves the original WDO algorithm.
\end{abstract}

\section{Introduction}

Nature always makes people produce a lot of inspiration. In the past, many natural heuristic algorithms have been proposed for solving real-world and large scale problems which are very difficult to solve by traditional methods, for example, genetic algorithm (GA) [1], particle swarm optimization (PSO) $[2,3]$, ant colony optimization (ACO) [4], differential evolution (DE) [5], firefly algorithm (FA) [6], bat algorithm (BA) [7], cuckoo search (CS) [8], flower pollination algorithm (FPA) [9], wind driven optimization (WDO) [10].

Because some heuristic algorithms are not very satisfactory in many respects, in the recent years, according to the characteristics of some algorithms, the heuristic algorithm combined with each other is an increasingly popular strategy to improve algorithms. These hybridizations have been shown to be effective global optimization algorithms and have been applied to solve application problems. For example, a hybrid of genetic algorithm (GA) and particle swarm optimization (PSO) is applied to recurrent neural/fuzzy network design [11]. A hybrid metaheuristic differential evolution (DE) and cuckoo search (CS) algorithm is implemented to solve the UCAV path planning problem [12]. A hybrid particle swarm optimization (PSO) and ant colony optimization (ACO) algorithm is implemented to solve hierarchical classification [13].

The wind driven optimization (WDO) is a novel natureinspired technique. It is a population based iterative heuristic process. The WDO was proposed by Bayraktar et al. in 2010 [10]. The inspiration of the WDO derives from the atmosphere, where wind blows in an attempt to balance the atmospheric pressure. Due to the fact that the WDO has few parameters in need of control and it is very easy to be carried out, it has been paid more attention by the academic community. But, in the early stage of solving optimization problems, the convergence speed of the WDO is quicker than others; when all the individuals are close to the optimal one in the late stage of solving optimization problems, it will lead to the loss of population diversity, and it is easy to fall into local optimum in finding better solutions.

In 1997, Storn and Price have introduced the DE algorithm [5]. In the next time, the DE algorithm has been applied to solve optimization problems in diverse fields. After the population initialization, the population through mutation, crossover, and selection operators generates new population, 


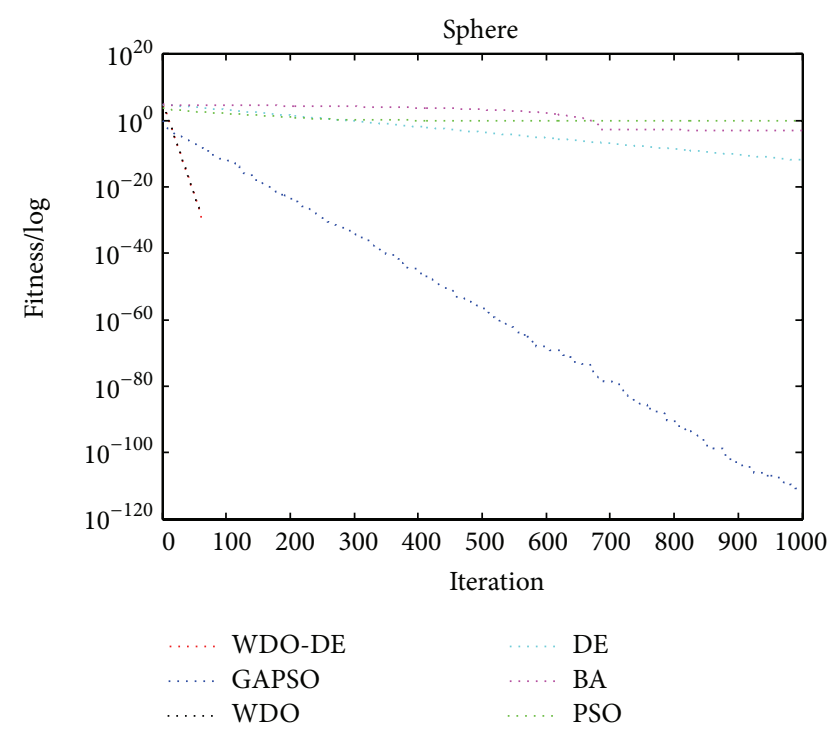

FIGURE 1: Comparison of performance of algorithms for minimization of $f_{1}(D=30)$.

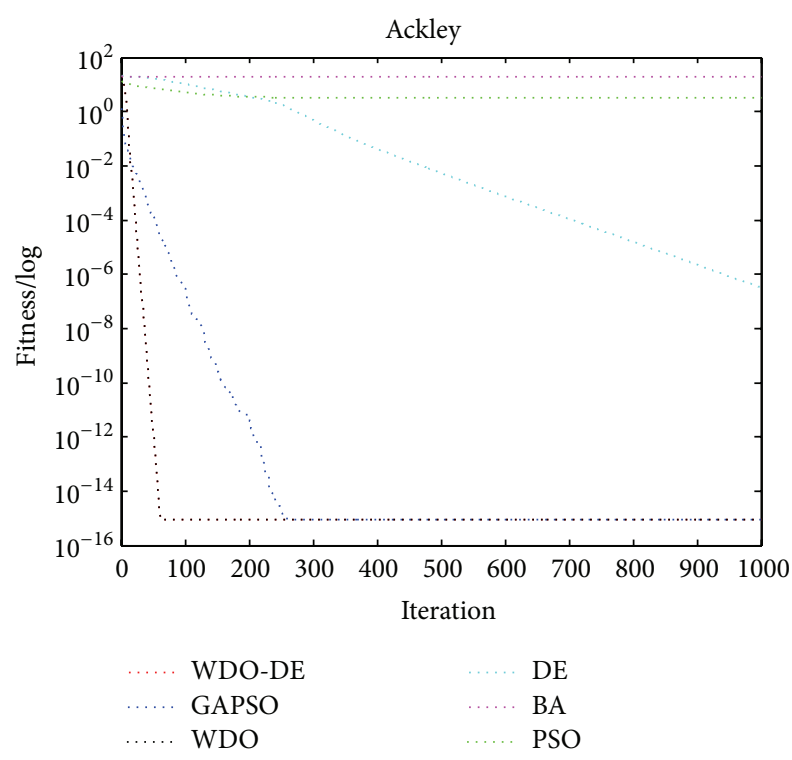

FIGURE 2: Comparison of performance of algorithms for minimization of $f_{2}(D=30)$.

it is able to maintain diversity of population, which can achieve the search of global optimal solution after several iterations. But it also has flaws. Its disadvantages contain the slow pace of convergence, and it is easy to fall into local optimum.

WDO algorithm can easily suffer from the premature convergence when solving global optimization problems. It is an important method for relieving the premature convergence to control the population diversity. In order to overcome the deficiencies of a single algorithm in solving a global optimization problem, in this paper, we propose a new hybrid global optimization algorithm based on the wind

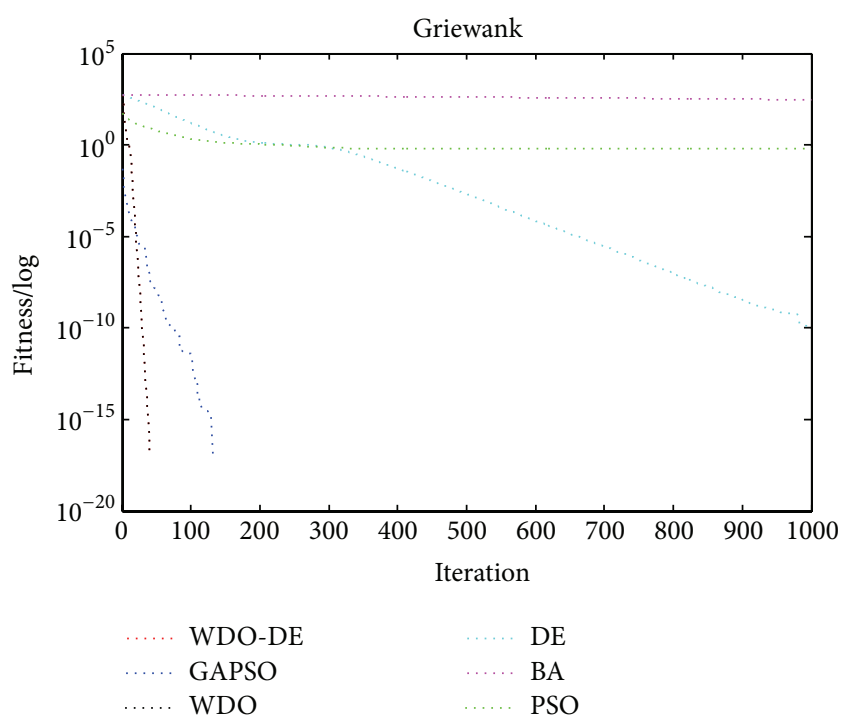

FIGURE 3: Comparison of performance of algorithms for minimization of $f_{3}(D=30)$.

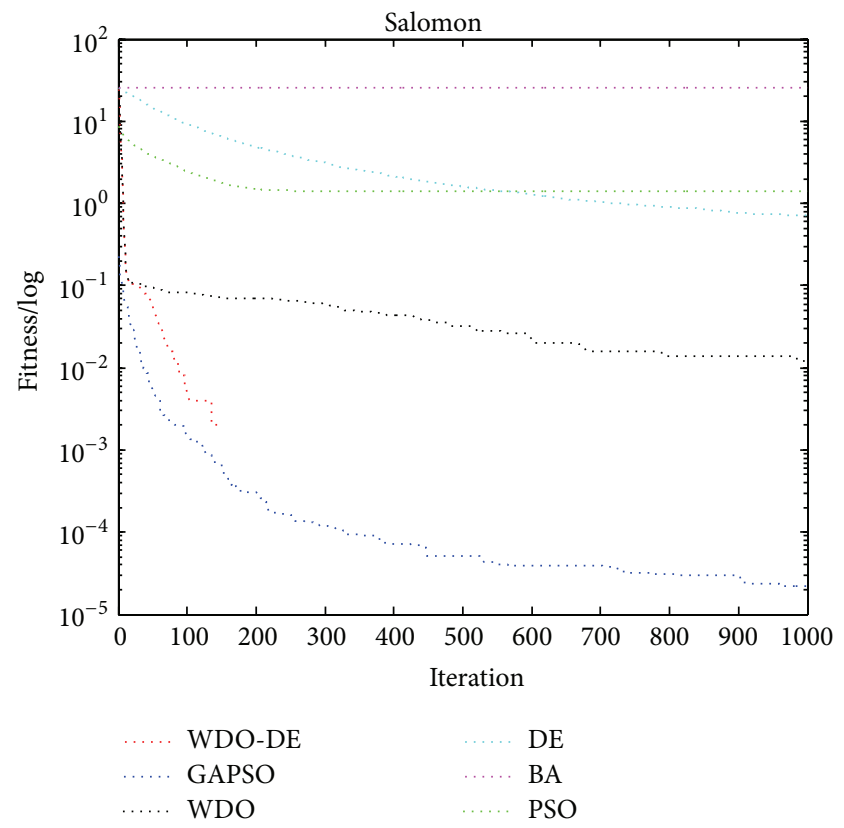

FIGURE 4: Comparison of performance of algorithms for minimization of $f_{4}(D=30)$.

driven optimization and differential evolution. This evolution strategy allows WDO and DE algorithms to give full play to their respective advantages. DE algorithm enables keeping the diversity of the population. This can be a good remedy defect of WDO algorithm, so it can avoid falling into a local optimum due to the loss of population diversity. And WDO algorithm converges faster; it can be good to make up for the shortcomings of DE algorithm in convergence speed, utilizing the individual of DE algorithm to guide the evolution of the individual of WDO algorithm, which reduces the risk of falling into a local optimal solution. It is not only to ensure 


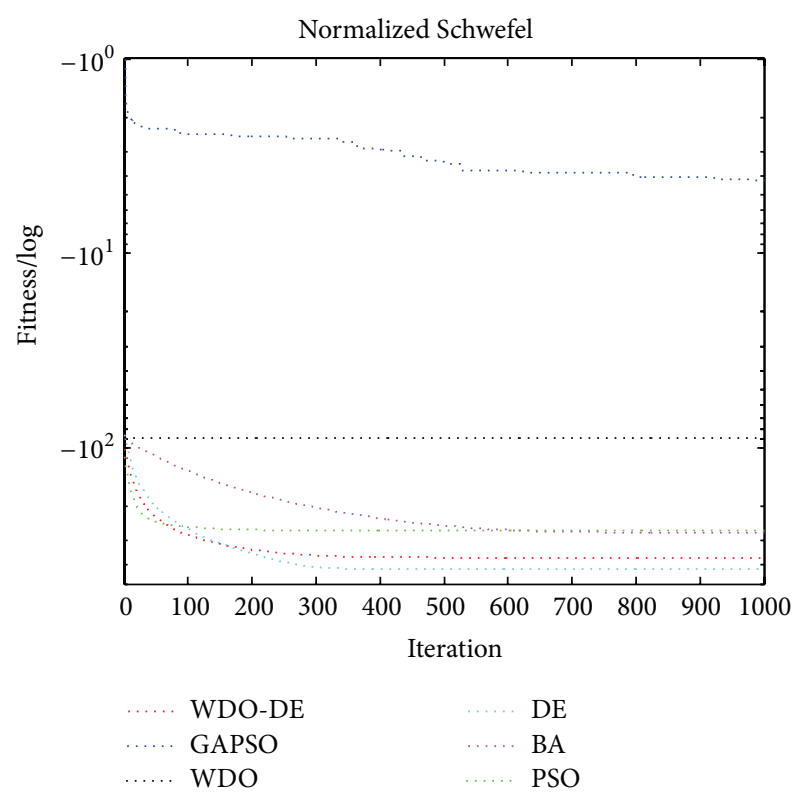

FIGURE 5: Comparison of performance of algorithms for minimization of $f_{5}(D=30)$.

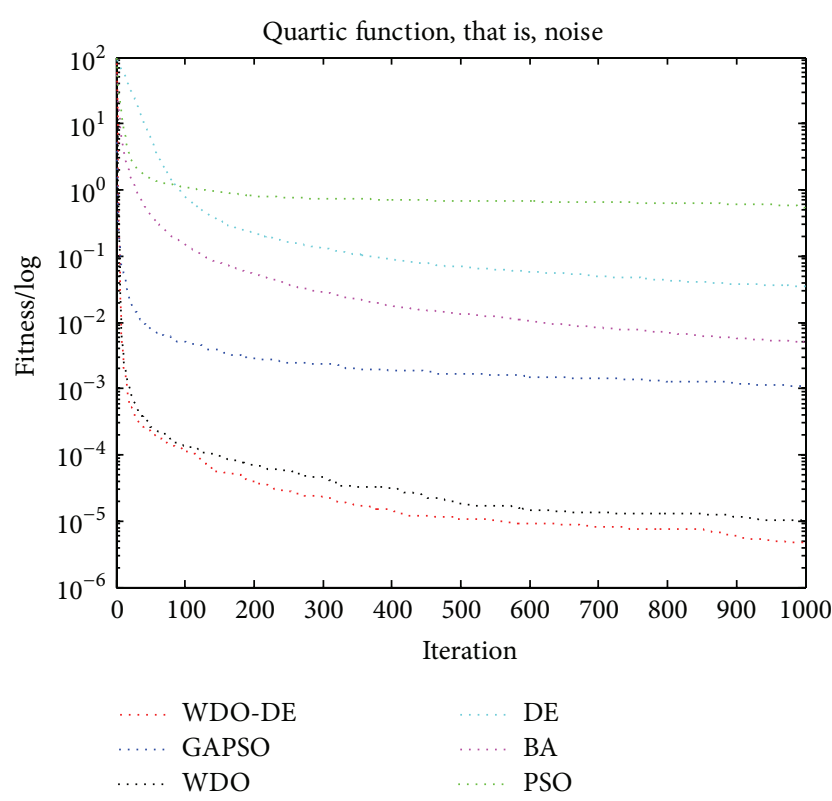

FIGURE 6: Comparison of performance of algorithms for minimization of $f_{6}(D=30)$.

the accuracy of the algorithm, but also to guarantee the speed of solving problems. Finally, the fifteen benchmark functions are tested; the experimental results show that the proposed algorithm can be feasible in both low-dimensional and highdimensional cases. Compared to GA-PSO, WDO, DE, PSO, and $\mathrm{BA}$ algorithm, the convergence speed and precision of WDO-DE are higher. This hybridization showed a better optimization performance and robustness and significantly improves the original WDO algorithm.

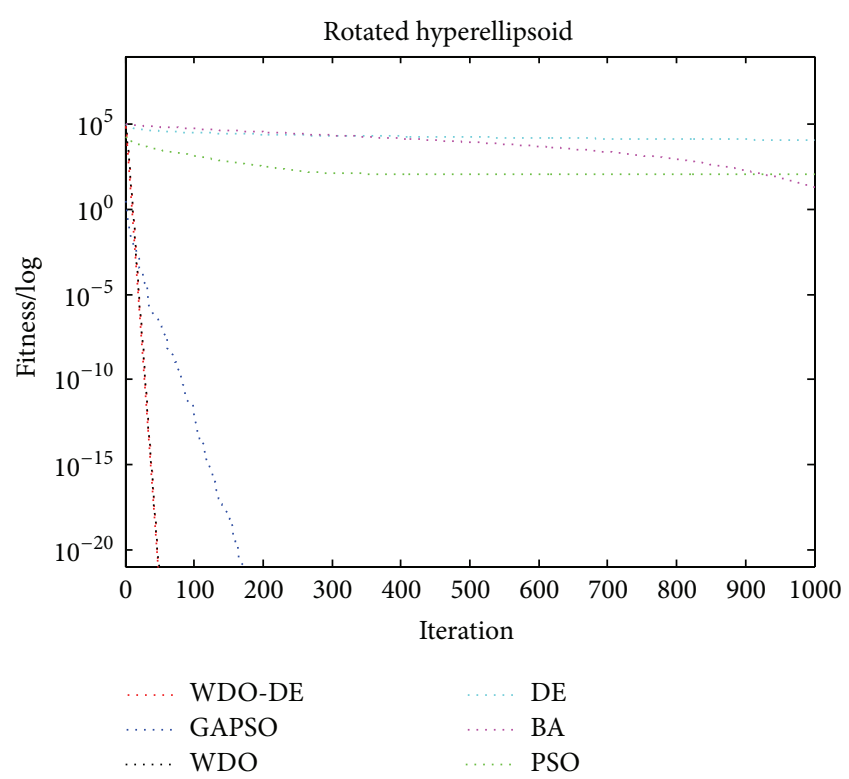

FIGURE 7: Comparison of performance of algorithms for minimization of $f_{7}(D=30)$.

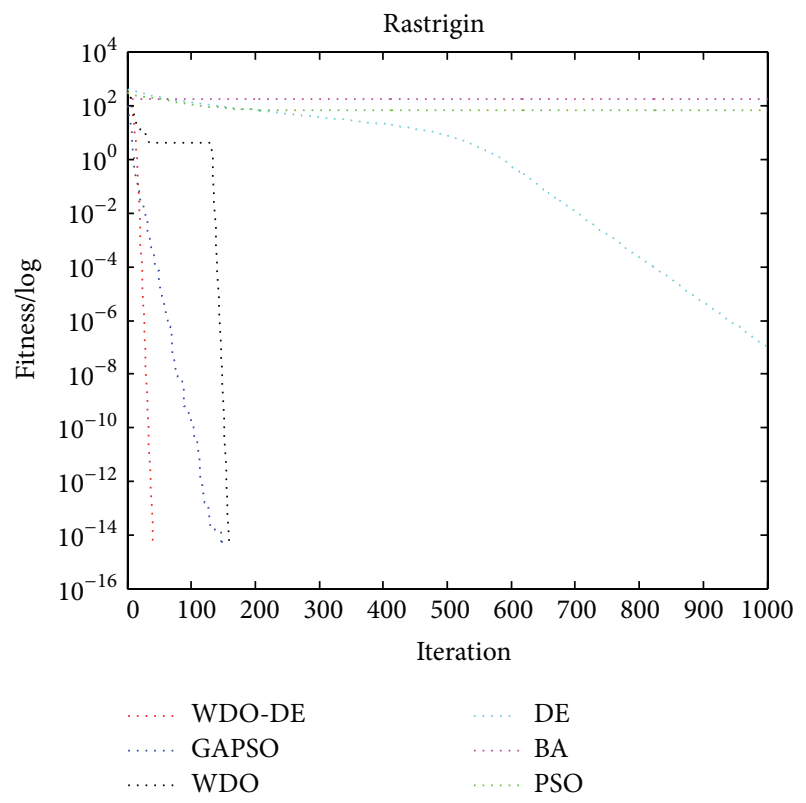

FIGURE 8: Comparison of performance of algorithms for minimization of $f_{8}(D=30)$.

\section{A Brief Introduction on WDO and DE Algorithm}

2.1. Wind Driven Optimization. The inspiration of the proposed WDO derives from the atmosphere. In the atmosphere, wind blows in an attempt to balance the imbalance of pressure. It flows from high pressure areas to low pressure areas at a velocity. Depending on the above analysis, some theoretical assumptions are formulated in derivation of the WDO algorithm. The starting point of WDO algorithm is 


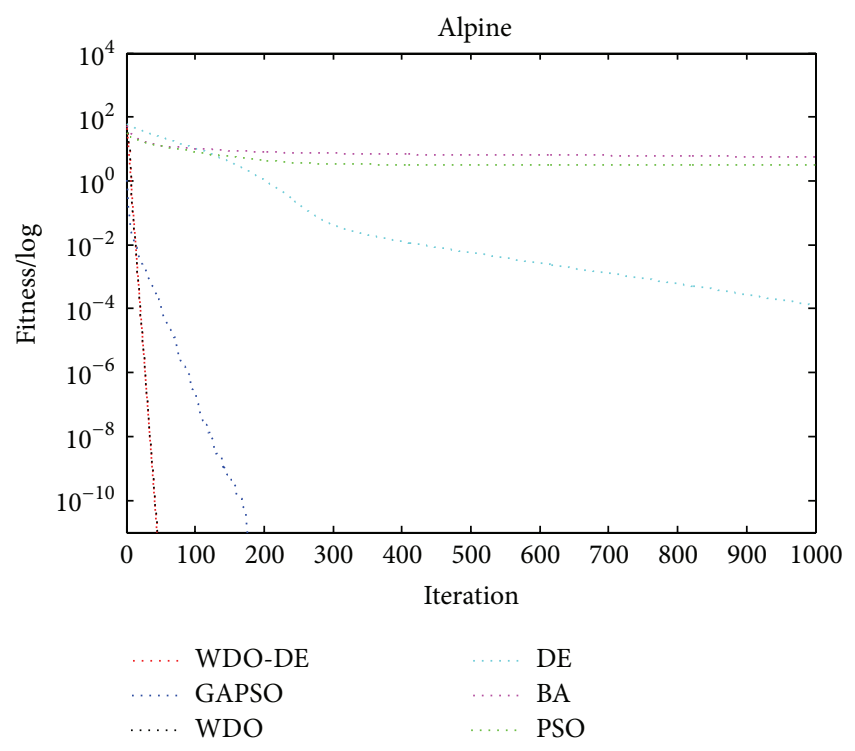

FIGURE 9: Comparison of performance of algorithms for minimization of $f_{9}(D=30)$.

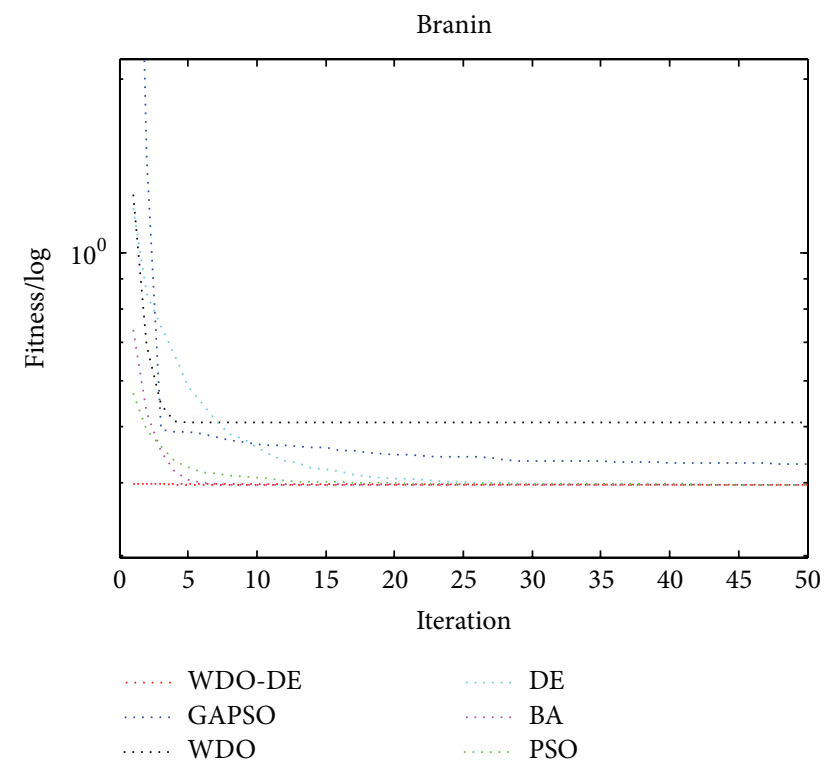

FIGURE 10: Comparison of performance of algorithms for minimization of $f_{10}(D=2)$.

Newton's second law of motion, which is used to provide accurate results for the analysis of atmospheric motion in the Lagrangian description $[14,15]$

$$
\rho \vec{\alpha}=\sum \vec{F}_{i}
$$

where $\vec{\alpha}$ is the acceleration, $\rho$ is the air density for an infinitesimal air parcel, and $\vec{F}_{i}$ are all the forces acting on the air parcel. In order to let air pressure establish the equation relationship with the air parcel's density and temperature, the ideal gas law is given by

$$
P=\rho R T
$$

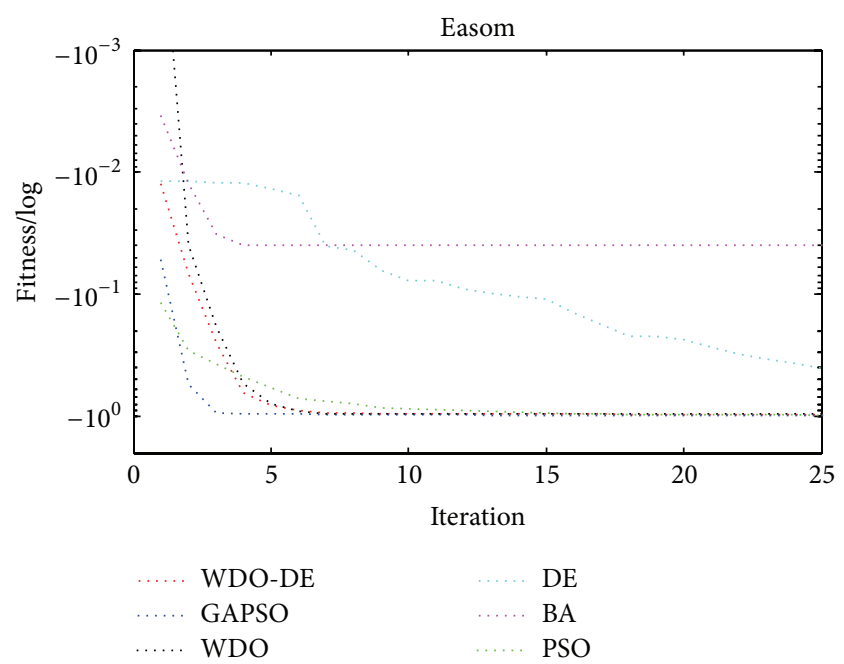

FIGURE 11: Comparison of performance of algorithms for minimization of $f_{11}(D=2)$.

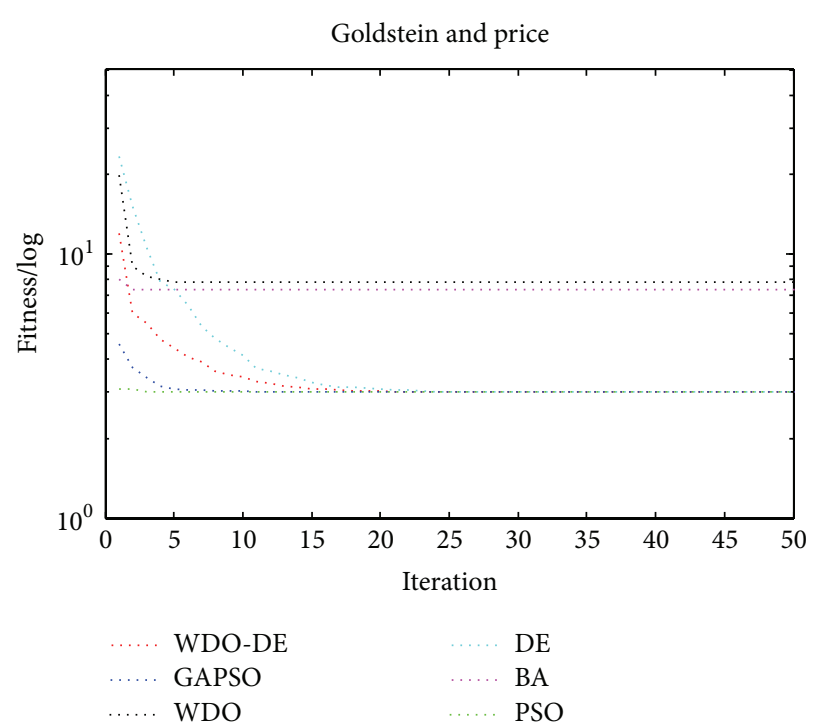

FIGURE 12: Comparison of performance of algorithms for minimization of $f_{12}(D=2)$.

where $P$ is the pressure, $R$ is the universal gas constant, and $T$ is the temperature.

The cause of the air movement is due to the combination of many forces, mainly including gravitational force $\left(\vec{F}_{G}\right)$, pressure gradient force $\left(\vec{F}_{\mathrm{PG}}\right)$, Coriolis force $\left(\vec{F}_{\mathrm{C}}\right)$, and friction force $\left(\vec{F}_{F}\right)$. The physical equations of the above mentioned forces are as follows:

$$
\begin{aligned}
\vec{F}_{G} & =\rho \delta V \vec{g}, \\
\vec{F}_{\mathrm{PG}} & =-\nabla P \delta V, \\
\vec{F}_{C} & =-2 \Omega \times \vec{u}, \\
\vec{F}_{F} & =-\rho \alpha \vec{u},
\end{aligned}
$$




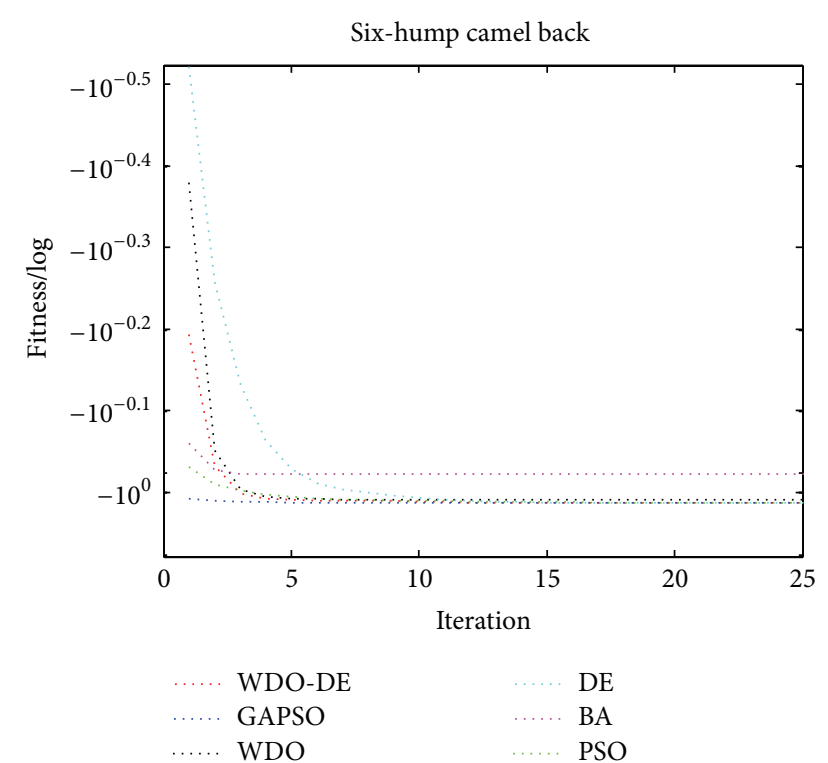

FIGURE 13: Comparison of performance of algorithms for minimization of $f_{13}(D=2)$.

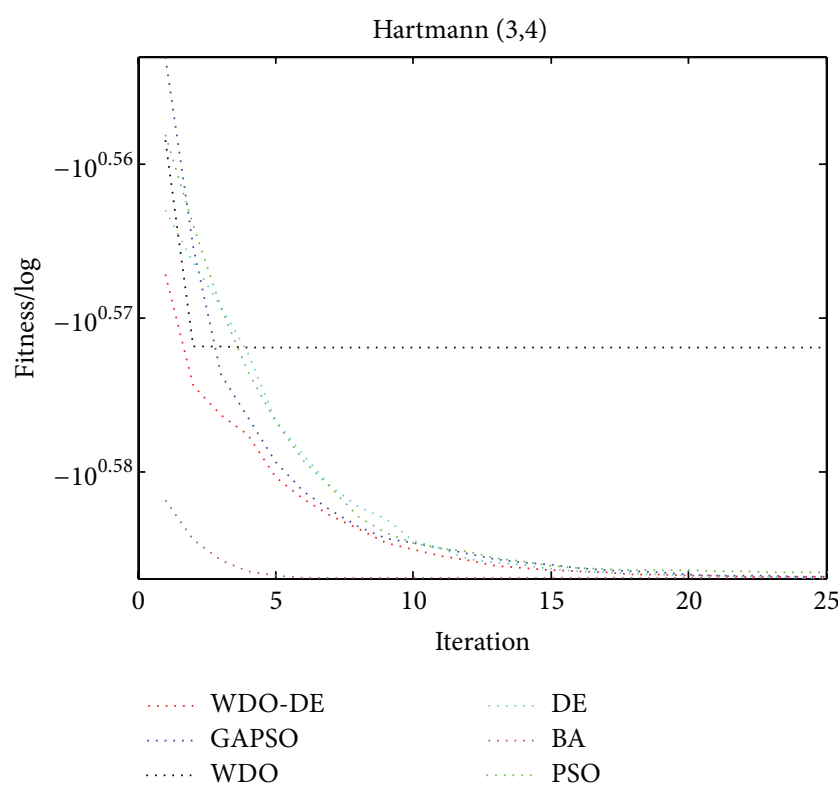

FIGURE 14: Comparison of performance of algorithms for minimization of $f_{14}(D=3)$.

where $\delta V$ is finite volume of the air, $\vec{g}$ represents the gravitational acceleration, $\nabla P$ represents the pressure gradient, $\Omega$ is rotation of the Earth, $\vec{u}$ represents the velocity vector of the wind, and $\alpha$ is the friction coefficient.

The forces mentioned above can be added to (1). The equation can be described as in

$$
\rho \frac{\Delta \vec{u}}{\Delta t}=(\rho \delta V \vec{g})+(-\nabla P \delta V)+(-2 \Omega \times \vec{u})+(-\rho \alpha \vec{u})
$$

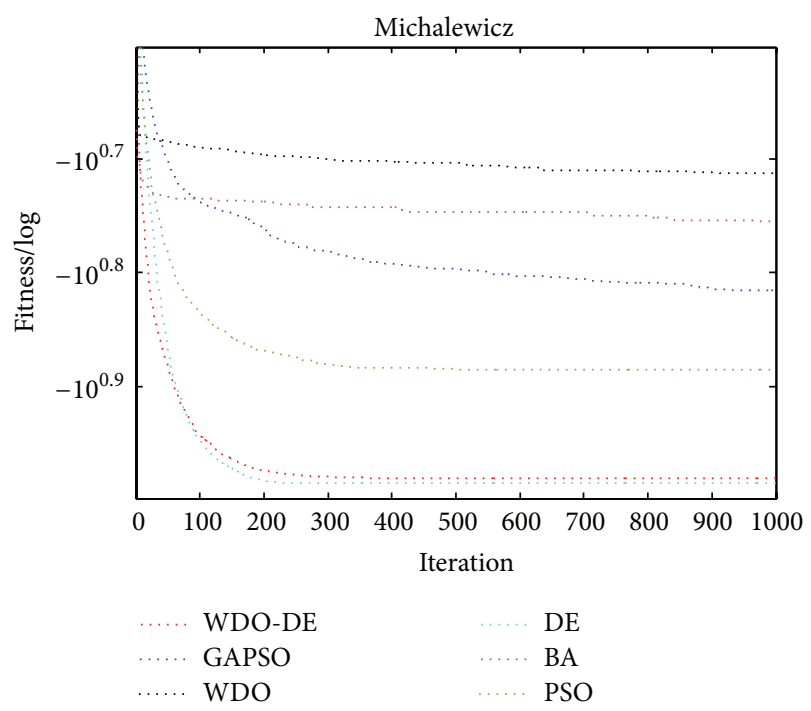

FIGURE 15: Comparison of performance of algorithms for minimization of $f_{15}(D=10)$.

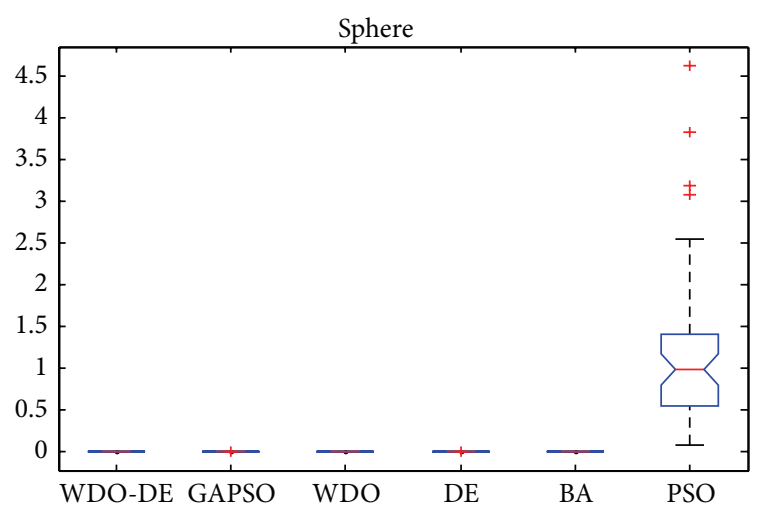

FIGURE 16: ANOVA tests of the global minimum values for $f_{1}(D=$ $30)$.

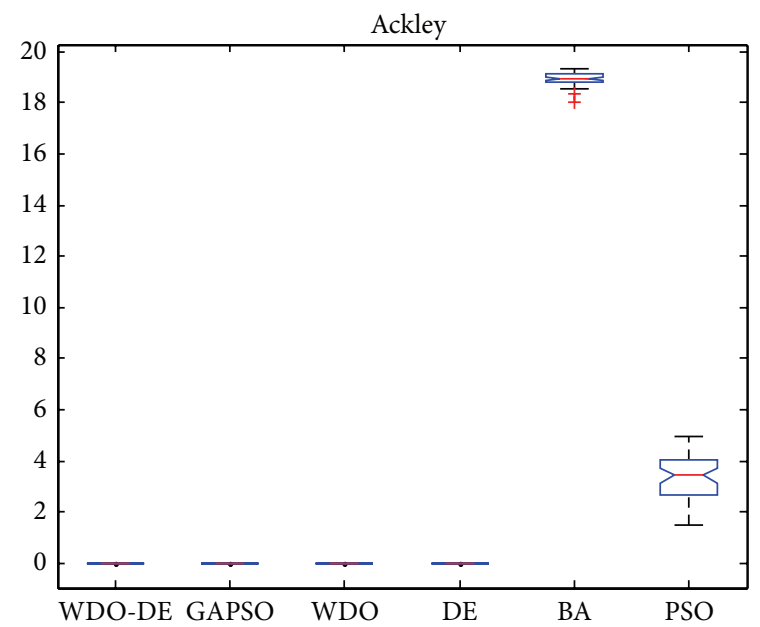

Figure 17: ANOVA tests of the global minimum values for $f_{2}(D=$ $30)$. 


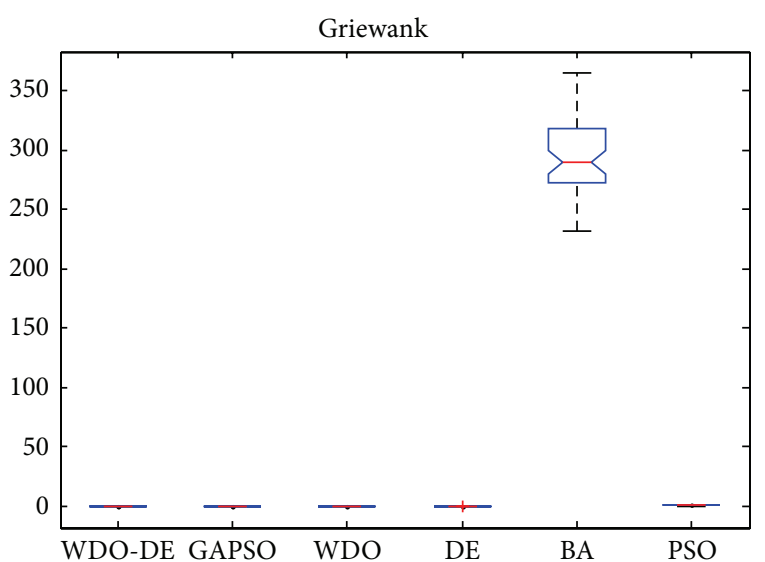

FIGURE 18: ANOVA tests of the global minimum values for $f_{3}(D=$ $30)$.

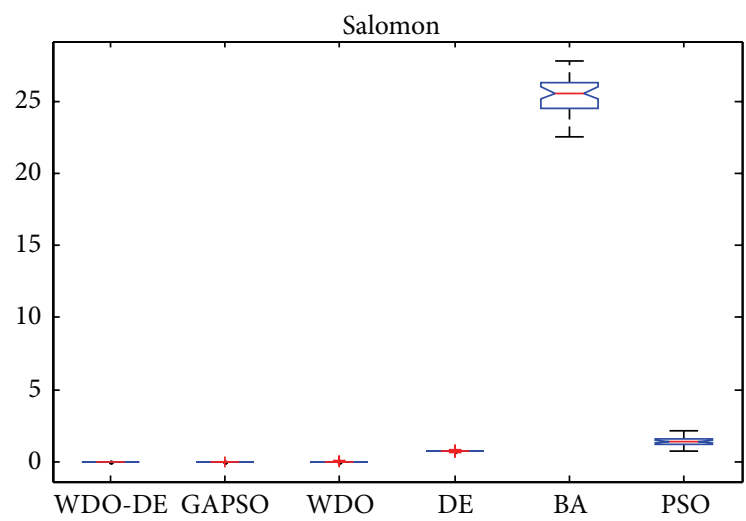

FIGURE 19: ANOVA tests of the global minimum values for $f_{4}(D=$ $30)$.

where the acceleration $\vec{\alpha}$ in (1) is rewritten as $\vec{\alpha}=\Delta \vec{u} / \Delta t$; for simplicity, set $\Delta t=1$; for an infinitesimal air parcel, set $\delta V=1$, which simplifies (4) to

$$
\rho \Delta \vec{u}=(\rho \vec{g})+(-\nabla P)+(-2 \Omega \times \vec{u})+(-\rho \alpha \vec{u}) .
$$

On the basis of (2), the density $\rho$ can be written in terms of the pressure; thus (5) can be rewritten as

$$
\Delta \vec{u}=\vec{g}+\left(-\nabla P \frac{R T}{P_{\text {cur }}}\right)+\left(\frac{-2 \Omega \times \vec{u} R T}{P_{\text {cur }}}\right)+(-\alpha \vec{u}),
$$

where $P_{\text {cur }}$ is the pressure of current location. It is assumed in the WDO algorithm that velocity and position of the air parcel are changing at each iteration. Thus, $\Delta \vec{u}$ can be written as $\Delta \vec{u}=\vec{u}_{\text {new }}-\vec{u}_{\text {cur }}$, where $\vec{u}_{\text {new }}$ represents the velocity in next iteration and $\vec{u}_{\text {cur }}$ is the velocity at the current iteration. $\vec{g}$ and $\nabla P$ are vectors, they can be broken down in direction and magnitude as $\vec{g}=|g|\left(0-x_{\text {cur }}\right),-\nabla P=\left|P_{\text {opt }}-P_{\text {cur }}\right|\left(x_{\text {opt }}-\right.$ $\left.x_{\text {cur }}\right), P_{\text {opt }}$ is the optimum pressure point that has been found so far, $x_{\text {opt }}$ is the optimum location that has been found so

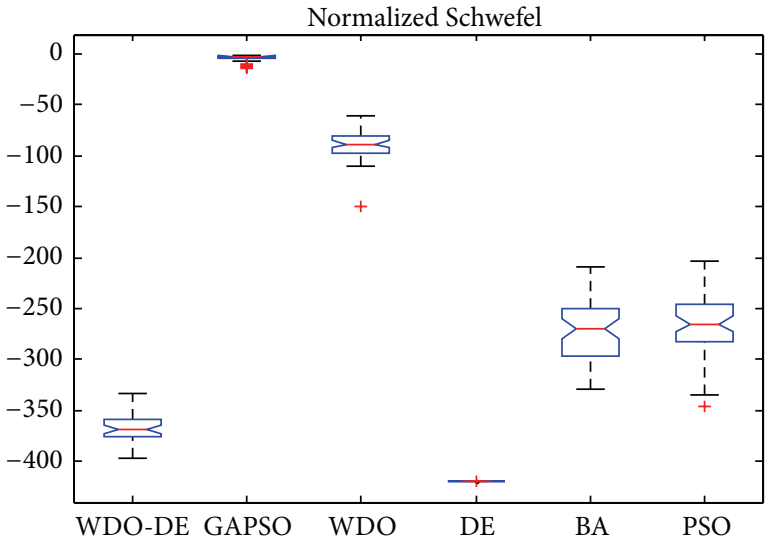

FIgURE 20: ANOVA tests of the global minimum values for $f_{5}(D=$ $30)$.

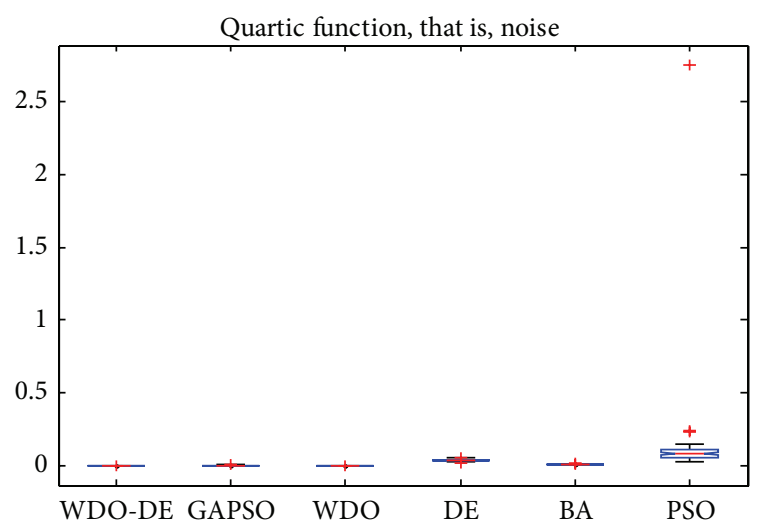

FIgURE 21: ANOVA tests of the global minimum values for $f_{6}(D=$ $30)$.

far, and $x_{\text {cur }}$ is the current location; updating (6) with the new equations, (6) can be rewritten as

$$
\begin{aligned}
\vec{u}_{\text {new }}= & (1-\alpha) \vec{u}_{\mathrm{cur}}-g x_{\mathrm{cur}} \\
& +\left(\frac{R T}{P_{\mathrm{cur}}}\left|P_{\mathrm{opt}}-P_{\mathrm{cur}}\right|\left(x_{\mathrm{opt}}-x_{\mathrm{cur}}\right)\right) \\
& +\left(\frac{-2 \Omega \times \vec{u} R T}{P_{\mathrm{cur}}}\right) .
\end{aligned}
$$

Finally, there are three additional substitutions needed. Firstly, the influence of the Coriolis force $(\Omega \times \vec{u})$ is replaced by the velocity influence from another dimension $\vec{u}_{\text {cur }}^{\text {other }}$. Secondly, all the coefficients are combined together; that is, $c=-2 R T$. Thirdly, in some cases where the pressure is extremely large, the updated velocities are too large to become meaningless and the efficiency of the WDO algorithm will be reduced. So the actual pressure value is replaced by rank among all air parcels based on their pressure values, the resulting equation of updating the velocity can be described 


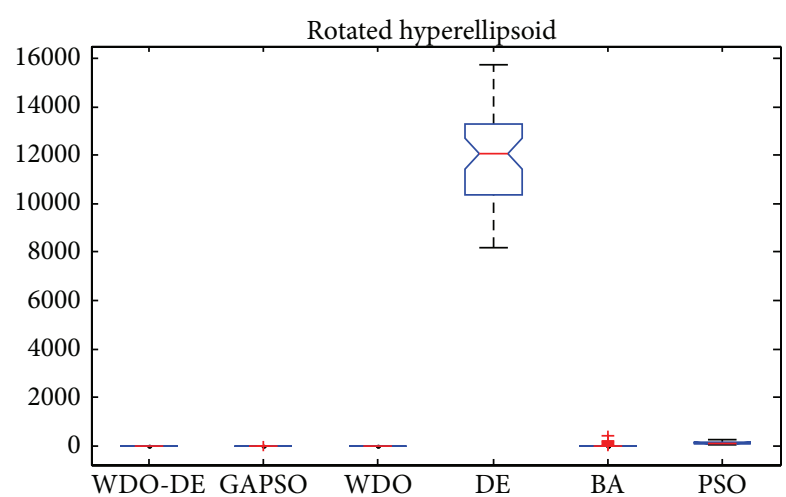

FIgURE 22: ANOVA tests of the global minimum values for $f_{7}(D=$ $30)$.

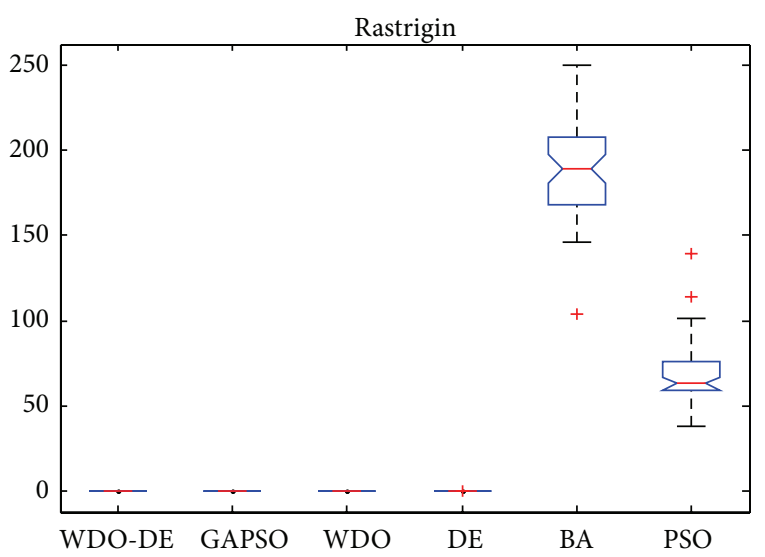

FIGURE 23: ANOVA tests of the global minimum values for $f_{8}(D=$ $30)$.

as in (8), and the equation of updating the location can be described as in (9):

$$
\begin{aligned}
\vec{u}_{\text {new }}= & (1-\alpha) \vec{u}_{\text {cur }}-g x_{\text {cur }} \\
& +\left(R T\left|1-\frac{1}{i}\right|\left(x_{\text {opt }}-x_{\text {cur }}\right)\right) \\
& +\left(\frac{c \vec{u}_{\text {cur }}^{\text {other dim }}}{i}\right), \\
\vec{x}_{\text {new }}= & \vec{x}_{\text {cur }}+\left(\vec{u}_{\text {new }} \times \Delta t\right),
\end{aligned}
$$

where $i$ is the ranking among all air parcels and $\vec{x}_{\text {new }}$ represents the new location for the next iteration.

WDO is similar to other nature-inspired optimization algorithms, but compared to other optimization algorithms, the code of WDO is more simple and easy to implement; it has less few control variables that need adjustment.

2.2. Differential Evolution. Differential evolution is introduced by Storn and Price in 1997 [5]. DE is an effective and simple global optimization algorithm.

First of all, a population is generated randomly, it may be represented as $X_{i, G}(i=1,2, \ldots, \mathrm{NP})=\left\{x_{i, G}^{1}, x_{i, G}^{2}, \ldots, x_{i, G}^{D}\right\}$,

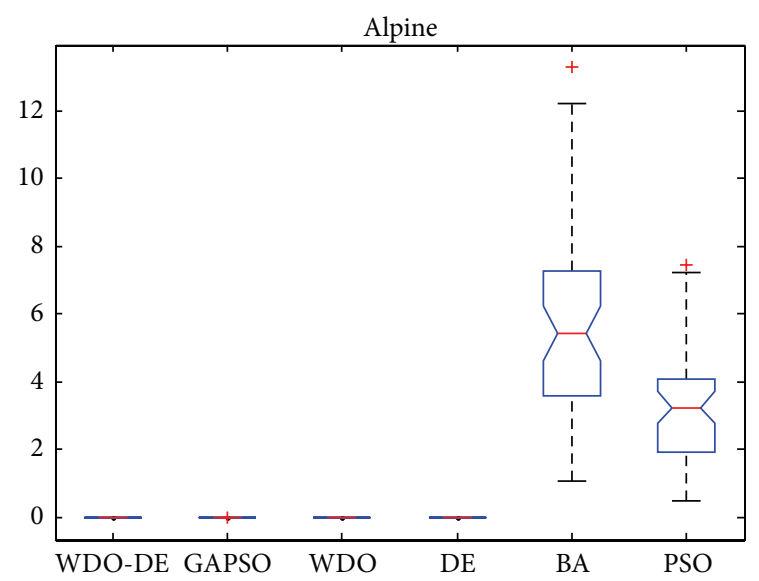

FIgURE 24: ANOVA tests of the global minimum values for $f_{9}(D=$ $30)$.

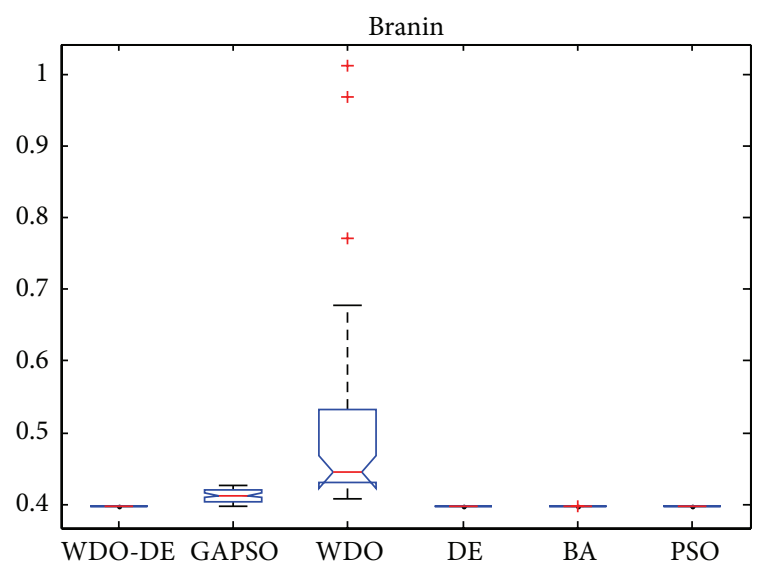

FIgURE 25: ANOVA tests of the global minimum values for $f_{10}(D=$ $30)$.

NP is the number of population, $D$ is the number of dimensions, and $G$ denotes the generation of the population. There are three operators-mutation, crossover, and selection. Then the original population will through three operators generate a new population. The main progress of DE in detail is as follows.

(1) Mutation. After initialization, mutation operation is used to generate the mutant vectors $V_{i, G}=\left\{v_{i, G}^{1}, v_{i, G}^{2}, \ldots, v_{i, G}^{D}\right\}$. Mutation operation usually has the following five most frequently implemented strategies.

$\mathrm{DE} / \mathrm{rand} / 1$ :

$$
V_{i, G}=X_{r_{1}^{i}, G}+F \cdot\left(X_{r_{2}^{i}, G}-X_{r_{3}^{i}, G}\right) .
$$

DE/best/1:

$$
V_{i, G}=X_{\text {best }, G}+F \cdot\left(X_{r_{1}^{i}, G}-X_{r_{2}^{i}, G}\right) .
$$

$\mathrm{DE} /$ current-to-best/1:

$$
\begin{aligned}
V_{i, G}= & X_{i, G}+F \cdot\left(X_{\text {best }, G}-X_{i, G}\right)+F \\
& \cdot\left(X_{r_{1}^{i}, G}-X_{r_{2}^{i}, G}\right) .
\end{aligned}
$$




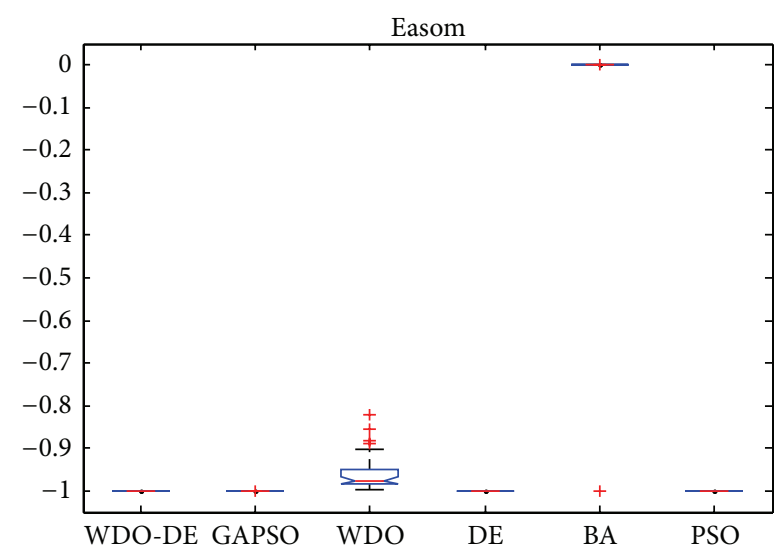

FigURE 26: ANOVA tests of the global minimum values for $f_{11}(D=$ 2).

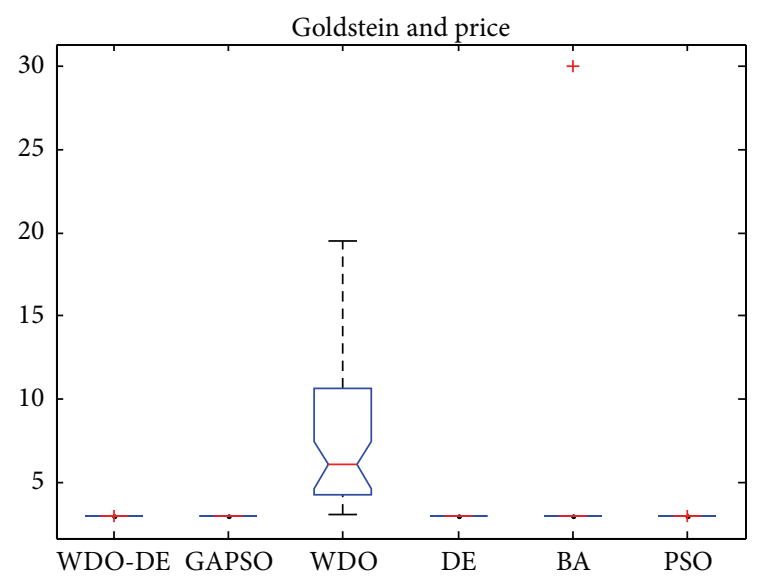

Figure 27: ANOVA tests of the global minimum values for $f_{12}(D=$ 2).

$\mathrm{DE} /$ best/2:

$$
\begin{aligned}
V_{i, G}= & X_{\text {best }, G}+F \cdot\left(X_{r_{1}^{i}, G}-X_{r_{2}^{i}, G}\right)+F \\
& \cdot\left(X_{r_{3}^{i}, G}-X_{r_{4}^{i}, G}\right) .
\end{aligned}
$$

$\mathrm{DE} / \mathrm{rand} / 2$ :

$$
\begin{aligned}
V_{i, G}= & X_{r_{1}^{i}, G}+F \cdot\left(X_{r_{2}^{i}, G}-X_{r_{3}^{i}, G}\right)+F \\
& \cdot\left(X_{r_{4}^{i}, G}-X_{r_{5}^{i}, G}\right) .
\end{aligned}
$$

The subscripts $r_{1}^{i}, r_{2}^{i}, r_{3}^{i}, r_{4}^{i}, r_{5}^{i}$ are mutually exclusive integers within the range $[1, \mathrm{NP}]$ and $F$ is the scale factor of difference vector.

(2) Crossover. After the mutation operation is completed, $\mathrm{DE}$ will utilize crossover operation to generate a trial vector $U_{i, G}=\left\{u_{i, G}^{1}, u_{i, G}^{2}, \ldots, u_{i, G}^{D}\right\}$ :

$$
u_{i, G}^{j}= \begin{cases}v_{i, G}^{j} & \text { if } \operatorname{rand}_{j}[0,1) \leq C R \text { or }\left(j=j_{\text {rand }}\right), \\ x_{i, G}^{j} & \text { others }\end{cases}
$$

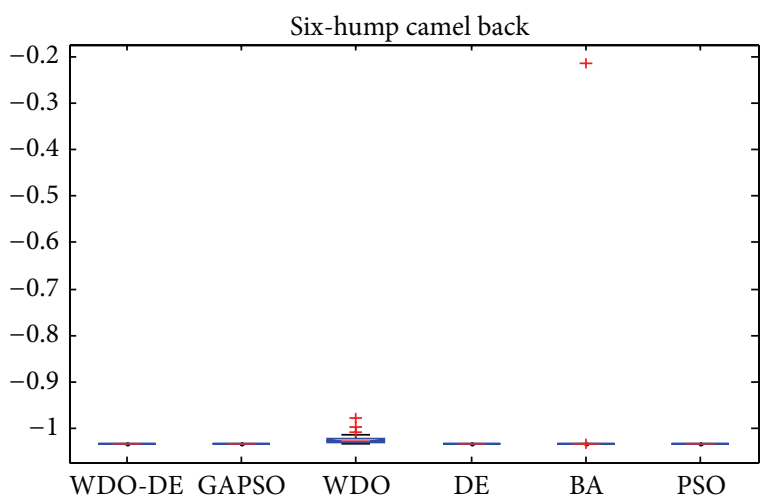

FIGURE 28: ANOVA tests of the globalminimum values for $f_{13}(D=$ 2).

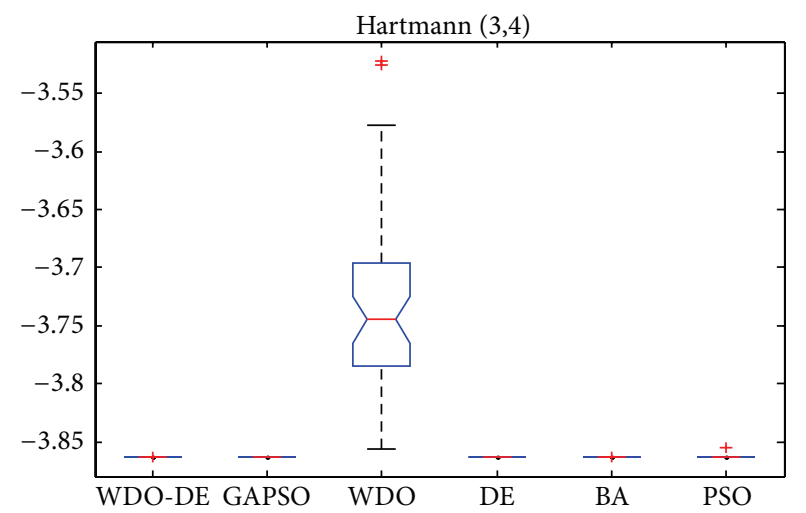

FIgURE 29: ANOVA tests of the global minimum values for $f_{14}(D=$ 2).

where $C R \in[0,1]$; it is a crossover constant. $j_{\text {rand }}$ is a random integer within the range $[1, D]$.

(3) Selection. After completing the first two operators, according the fitness value of trial vectors, DE utilizes selection operation to select the best one for the next generation:

$$
X_{i, G+1}= \begin{cases}U_{i, G} & \text { if } f\left(U_{i, G}\right) \leq f\left(X_{i, G}\right), \\ X_{i, G} & \text { otherwise. }\end{cases}
$$

After gradual iteration, DE can achieve the search of global optimal solution.

\section{WDO-DE Algorithm}

This section focuses on the rationale of the WDO-DE algorithm. WDO and DE are based on the population of the global search techniques. And the WDO-DE is based on a double population evolution strategy [16]. The individuals both in WDO and DE employ an information sharing mechanism to implement coevolution. The strategy makes WDO-DE enjoy the advantages of two algorithms. It can maintain diversity of the populations, and the WDO-DE algorithm has the capability to jump out of the local optimal 
Step 1. Initialize parameters.

$N$ (Population size); $G$ (Max number of generations);

Parameters of WDO: RT (RT coefficient); $\alpha$ (The friction coefficient); max $V$ (Maximum allowed speed); $g$ (Gravitational constant); $c$ (Constant in the update equation).

Parameters of DE: F (Mutation scale factor); Pc (Crossover probability).

Step 2. Initialization populations.

Step 2.1. Generate one initial population with $N / 2$ air particles, each air particle assign random location $P_{i, G}^{\text {wdo }}$ and velocity

$V_{i, G}^{\text {wdo }}$, evaluation the population and identify the best solution of WDO algorithm $P_{\text {best }, G}^{\text {wdo }}$;

Step 2.2. Generate one initial population $P_{i, G}^{\mathrm{de}}$ with $N / 2$ individuals, evaluation the population and identify the best solution $P_{\text {best }, \text { de }}^{\text {. }}$.

Step 3. Identify the best solution $P_{\text {best }}$ among all particles in WDO and DE.

Step 4. While stopping criterion is not satisfied

Step 4.1. Running process of the WDO algorithm

for $i=1$ to the $N / 2$ do

$P_{\text {best }, G}^{\text {wdo }}=P_{\text {best }}$

Generate the trial velocity according to (8)

Generate the trial location $U_{i, G}$ by (9)

Evaluate the trial location $U_{i, G}$

$$
\begin{aligned}
& \text { If } f\left(U_{i, G}\right) \leq f\left(P_{i, G}^{\text {wdo }}\right) \\
& \quad P_{i, G+1}^{\text {wdo }}=U_{i, G}, \quad f\left(P_{i, G+1}^{\text {wdo }}\right)=f\left(U_{i, G}\right) \\
& \text { If } f\left(U_{i, G}\right) \leq f\left(P_{\text {best }, G}^{\text {wdo }}\right) \\
& \quad P_{\text {best, }, G}^{\text {wdo }}=U_{i, G}, \quad f\left(P_{\text {best }, G}^{\text {wdo }}\right)=f\left(U_{i, G}\right) \\
& \text { End if } \\
& \text { End if }
\end{aligned}
$$

End for

Step 4.2. Running process of the DE algorithm

for $i=1$ to the $N / 2$ do

$$
\begin{aligned}
& P_{\text {best,G }}^{\text {de }}=P_{\text {best }} \\
& \text { Generate } V_{i, G} \text { using (11) }
\end{aligned}
$$

Generate the trial vector $U_{i, G}$ by (15)

Evaluate the trial vector $U_{i, G}$

$$
\begin{aligned}
& \text { If } f\left(U_{i, G}\right) \leq f\left(P_{i, G}^{\mathrm{de}}\right) \\
& \quad P_{i, G+1}^{\mathrm{de}}=U_{i, G}, \quad f\left(P_{i, G+1}^{\mathrm{de}}\right)=f\left(U_{i, G}\right) \\
& \quad \text { If } f\left(U_{i, G}\right) \leq f\left(P_{\mathrm{best}, G}^{\mathrm{de}}\right) \\
& \quad P_{\text {best, },}^{\mathrm{de}}=U_{i, G}, \quad f\left(P_{\text {best }, G}^{\mathrm{de}}\right)=f\left(U_{i, G}\right) \\
& \text { End if } \\
& \text { End if }
\end{aligned}
$$

End for

Step 4.3. Identify the best solution $P_{\text {best }}$

$$
\text { If } \begin{array}{r}
f\left(P_{\text {best }, G}^{\text {wdo }}\right) \leq f\left(P_{\text {best }, G}^{\mathrm{de}}\right) \\
P_{\text {best }}=P_{\text {best }, G}^{\text {wdo }}
\end{array}
$$

Else

End if

$$
P_{\text {best }}=P_{\text {best }, G}^{\mathrm{de}}
$$

Step 4.4. Increment the generation count $G=G+1$

Step 5. End while

Algorithm 1: WDO-DE.

solution. Based on the above description, the main procedure of WDO-DE is as shown in Algorithm 1.

\section{Experimental Results}

4.1. Experimental Setup. All algorithms are implemented in MATLAB R2012a, and experiments are performed on a Pentium $3.00 \mathrm{GHz}$ Processor with $4.0 \mathrm{~GB}$ of memory, Windows 7 operating system.
4.2. Benchmark Test Functions. To test the performance of WDO-DE algorithm, we use 15 benchmark functions $[17,18]$ which have been widely used in the test. Among these benchmarks, part I contains the nine high-dimensional functions and part II contains six low-dimensional functions. Table 1 has shown the benchmark functions.

4.3. Parameters Setting. In this section, the parameters setting are presented. Bayraktar et al. did a lot of research 


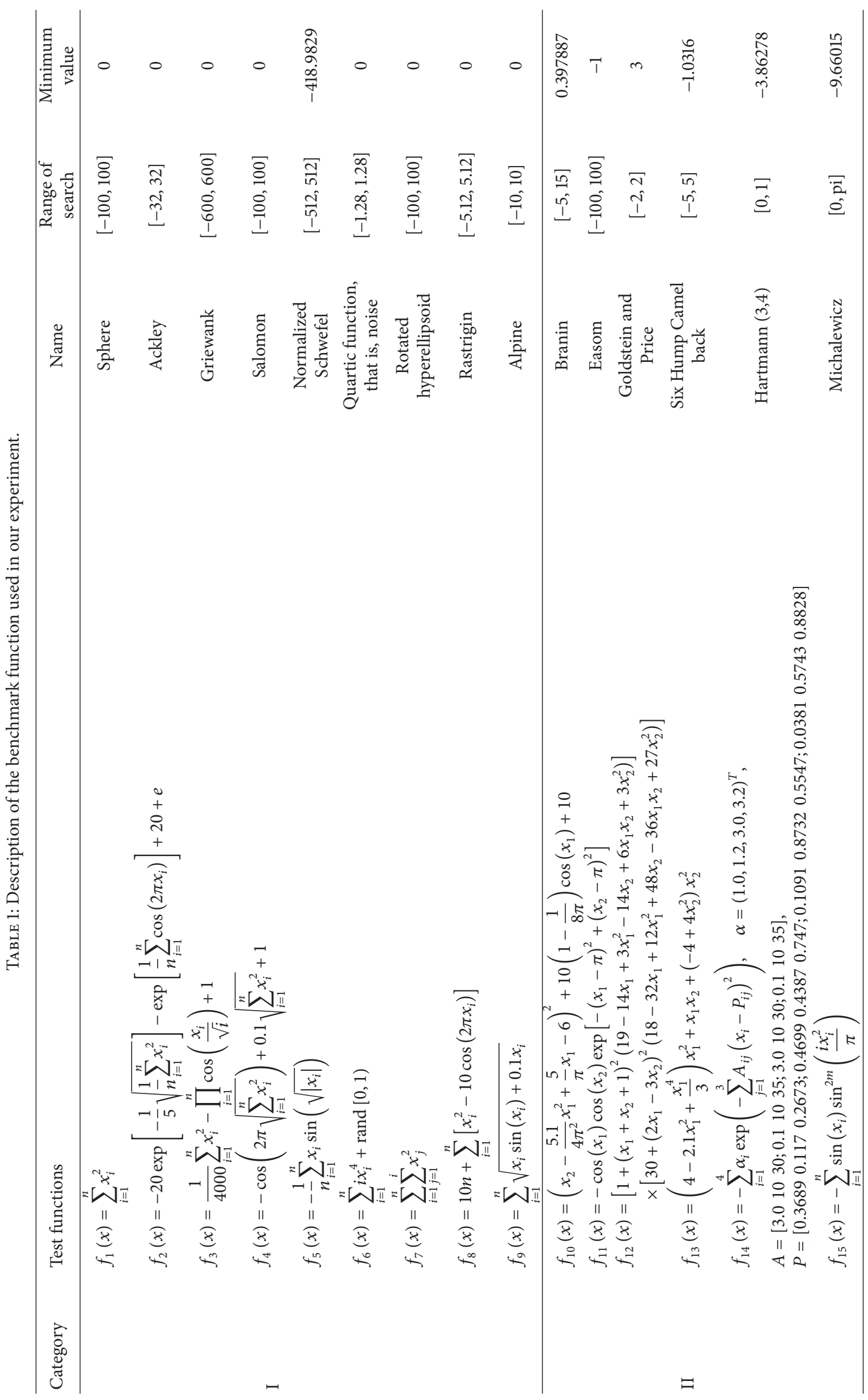




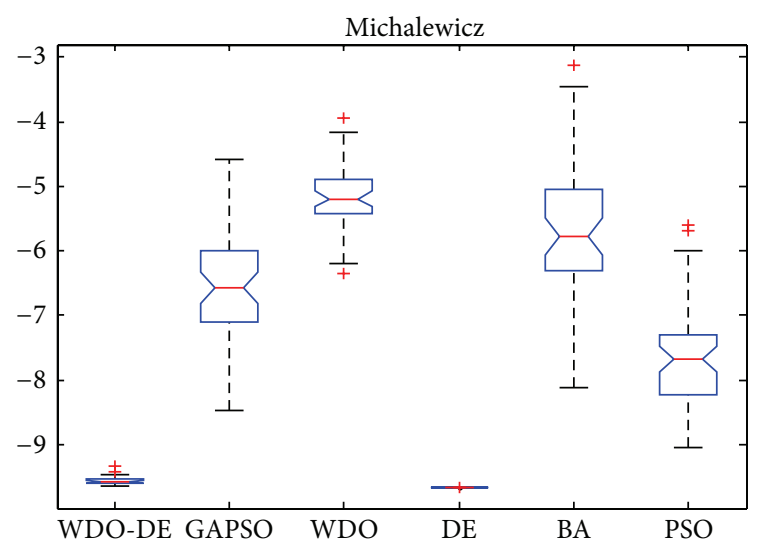

Figure 30: ANOVA tests of the global minimum values for $f_{15}(D=$ 3).

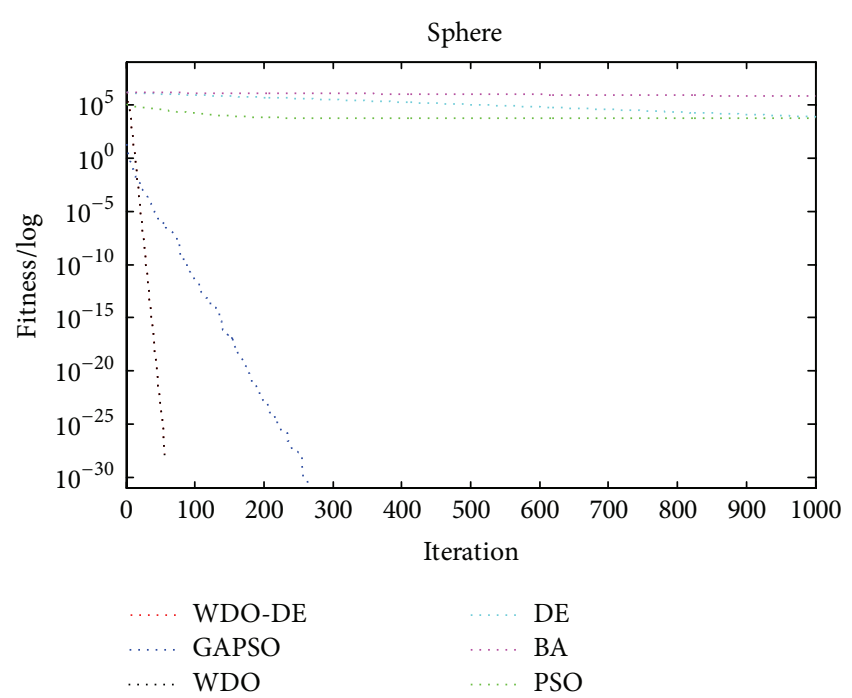

FIGURE 31: Comparison of performance of algorithms for minimization of $f_{1}(D=500)$.

TABLE 2: The parameters set of WDO.

\begin{tabular}{lc}
\hline Parameters & Value \\
\hline Population size & 50 \\
Max number of iterations & 1000 \\
RT coefficient & 1 \\
Constants in the update equation & 0.8 \\
Maximum allowed speed & 0.3 \\
Gravitational constant & 0.6 \\
Coriolis effect & 0.7 \\
\hline
\end{tabular}

for the parameters set of WDO [19]. The parameters set of $\mathrm{WDO}$ and DE is based on the practical experience to take the appropriate value. Tables 2 and 3 represent the necessary parameters in our experiment [20].

4.4. Algorithm Performance Comparison. In this section, in order to test the performance of WDO-DE algorithm,

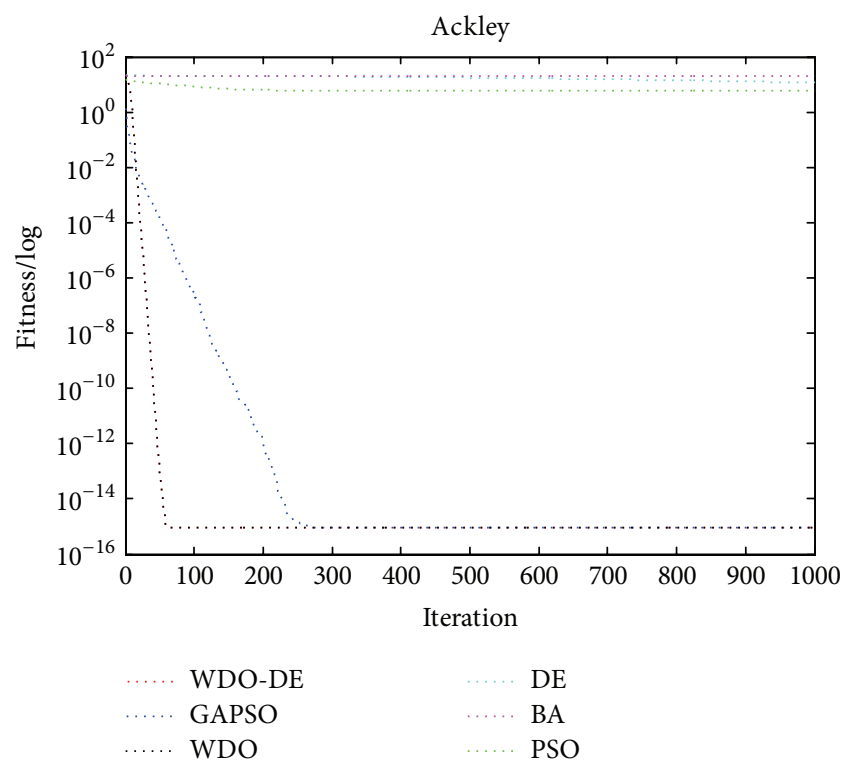

FIGURE 32: Comparison of performance of algorithms for minimization of $f_{2}(D=1000)$.

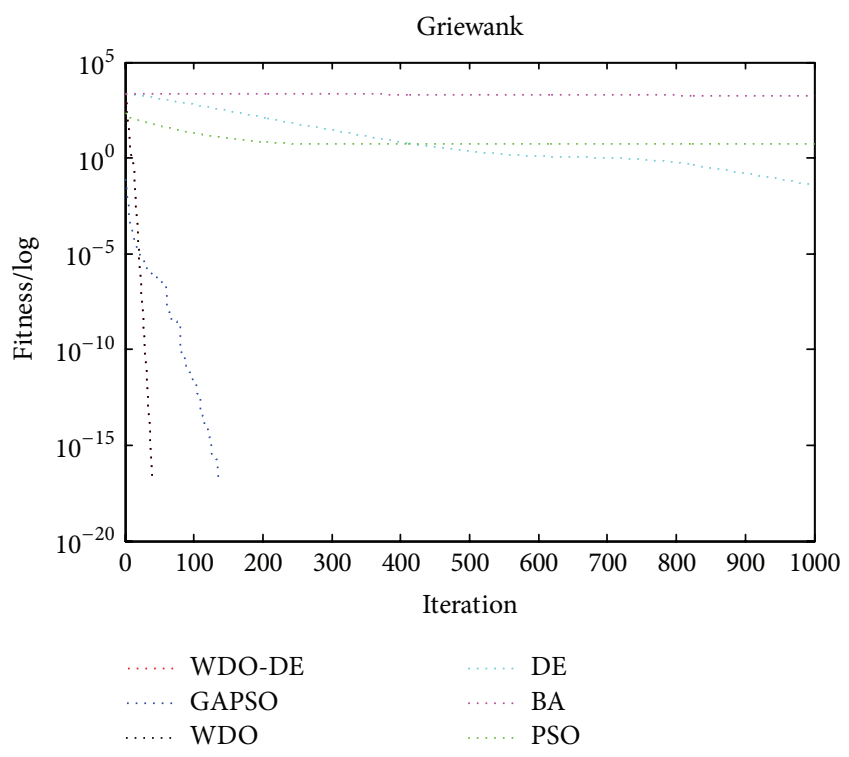

FIgURE 33: Comparison of performance of algorithms for minimization of $f_{3}(D=100)$.

TABLE 3: The parameters set of DE.

\begin{tabular}{lc}
\hline Parameters & Value \\
\hline Population size & 50 \\
Max number of iterations & 1000 \\
Mutation scale factor & 0.2 \\
Crossover probability & 0.03 \\
\hline
\end{tabular}

WDO-DE algorithm has been compared with the algorithms GA-PSO, WDO, DE, BA, and PSO in low dimension and high dimension. The mean results, standard deviation (Std.) 
TABLE 4: Comparison of performance of algorithms in low dimension.

\begin{tabular}{|c|c|c|c|c|c|c|c|}
\hline \multirow{2}{*}{$\begin{array}{l}\text { Test } \\
\text { functions }\end{array}$} & \multirow{2}{*}{ Result } & \multicolumn{6}{|c|}{ Algorithm } \\
\hline & & PSO & BA & $\mathrm{DE}$ & WDO & GAPSO & WDO-DE \\
\hline \multirow{5}{*}{$\begin{array}{l}f_{1} \\
(D=30)\end{array}$} & Mean & 1.198565069 & 0.001259031 & $1.15546 E-12$ & 0 & $8.938 E-112$ & 0 \\
\hline & Std. & 0.986982066 & 0.000164979 & $3.96904 E-13$ & 0 & $6.2926 E-111$ & $\mathbf{0}$ \\
\hline & Worst & 4.623331223 & 0.001579358 & $2.31909 E-12$ & 0 & $4.4499 E-110$ & $\mathbf{0}$ \\
\hline & Best & 0.077133013 & 0.000941037 & $6.51508 E-13$ & 0 & $1.7446 E-133$ & $\mathbf{0}$ \\
\hline & Rank & 6 & 5 & 4 & 1 & 3 & 1 \\
\hline \multirow{5}{*}{$\begin{array}{l}f_{2} \\
(D=30)\end{array}$} & Mean & 3.23151972 & 18.95562594 & $3.29757 E-07$ & $8.88178 E-16$ & $8.88178 E-16$ & $8.88178 E-16$ \\
\hline & Std. & 0.928231321 & 0.240934834 & $4.98931 E-08$ & 0 & 0 & 0 \\
\hline & Worst & 4.958505686 & 19.31856935 & $4.15132 E-07$ & $8.88178 E-16$ & $8.88178 E-16$ & $8.88178 E-16$ \\
\hline & Best & 1.51521671 & 18.06057448 & $2.41907 E-07$ & $8.88178 E-16$ & $8.88178 E-16$ & $8.88178 E-16$ \\
\hline & Rank & 5 & 6 & 5 & 1 & 1 & 1 \\
\hline \multirow{5}{*}{$\begin{array}{l}f_{3} \\
(D=30)\end{array}$} & Mean & 0.617836326 & 293.8529401 & $9.92471 E-11$ & 0 & 0 & 0 \\
\hline & Std. & 0.243522453 & 31.91402142 & $2.04264 E-10$ & 0 & 0 & $\mathbf{0}$ \\
\hline & Worst & 1.060834843 & 364.9175036 & $1.24088 E-09$ & 0 & 0 & $\mathbf{0}$ \\
\hline & Best & 0.118835584 & 231.9851842 & $6.60083 E-12$ & 0 & 0 & $\mathbf{0}$ \\
\hline & Rank & 5 & 6 & 4 & 1 & 1 & 1 \\
\hline \multirow{5}{*}{$\begin{array}{l}f_{4} \\
(D=30)\end{array}$} & Mean & 1.399873346 & 25.41987335 & 0.703241831 & 0.011986233 & $2.22495 E-05$ & 0 \\
\hline & Std. & 0.257935287 & 1.292363757 & 0.060062328 & 0.032788391 & $2.93452 E-05$ & $\mathbf{0}$ \\
\hline & Worst & 2.099873346 & 27.79987335 & 0.800431238 & 0.099920546 & 0.000122472 & $\mathbf{0}$ \\
\hline & Best & 0.699873346 & 22.59987335 & 0.599891695 & 0 & 0 & $\mathbf{0}$ \\
\hline & Rank & 5 & 6 & 4 & 3 & 2 & 1 \\
\hline \multirow{5}{*}{$\begin{array}{l}f_{5} \\
(D=30)\end{array}$} & Mean & -265.951733 & -272.200713 & -418.982887 & -89.1395986 & -4.234983 & -366.236378 \\
\hline & Std. & 30.56577124 & 27.19605457 & $8.05405 E-12$ & 14.48624741 & 3.304955999 & 12.75651852 \\
\hline & Worst & -202.664961 & -208.394008 & -418.982887 & -60.8635682 & -1.84114041 & -332.945962 \\
\hline & Best & -346.349165 & -328.828387 & -418.982887 & -149.39382 & -14.3267589 & -397.157289 \\
\hline & Rank & 4 & 3 & 1 & 5 & 6 & 2 \\
\hline \multirow{5}{*}{$\begin{array}{l}f_{6} \\
(D=30)\end{array}$} & Mean & $1.39 E-01$ & 0.005067 & 0.035133193 & $1.02406 E-05$ & 0.001098329 & $4.81416 \mathrm{E}-06$ \\
\hline & Std. & $3.80 E-01$ & 0.001999654 & 0.006615916 & $1.03384 E-05$ & 0.000919425 & $5.23045 \mathrm{E}-06$ \\
\hline & Worst & $2.75 E+00$ & 0.013185707 & 0.053066164 & $4.44353 E-05$ & 0.004461194 & $2.57286 \mathrm{E}-05$ \\
\hline & Best & $2.30 E-02$ & 0.002422956 & 0.019201609 & $7.08496 E-08$ & $2.49604 E-05$ & $6.73402 E-08$ \\
\hline & Rank & 6 & 4 & 5 & 2 & 3 & 1 \\
\hline \multirow{5}{*}{$\begin{array}{l}f_{7} \\
(D=30)\end{array}$} & Mean & 120.2398214 & 20.32158962 & 11826.12725 & 0 & $1.5811 E-114$ & $\mathbf{0}$ \\
\hline & Std. & 71.10080897 & 67.06165947 & 1857.70219 & 0 & $1.0078 E-113$ & 0 \\
\hline & Worst & 273.1473491 & 408.7930042 & 15721.31651 & 0 & $7.097 E-113$ & 0 \\
\hline & Best & 20.21367214 & 0.004650193 & 8201.656294 & 0 & $6.0955 E-138$ & $\mathbf{0}$ \\
\hline & Rank & 5 & 4 & 6 & 1 & 3 & 1 \\
\hline \multirow{5}{*}{$\begin{array}{l}f_{8} \\
(D=30)\end{array}$} & Mean & 68.83078526 & 188.2789504 & $9.98004 E-08$ & 0 & 0 & 0 \\
\hline & Std. & 18.36191672 & 28.50077605 & $1.1162 E-07$ & 0 & 0 & $\mathbf{0}$ \\
\hline & Worst & 139.4625725 & 249.9566 & $6.78839 E-07$ & 0 & 0 & 0 \\
\hline & Best & 38.07588886 & 103.6807268 & $1.22403 E-08$ & 0 & 0 & $\mathbf{0}$ \\
\hline & Rank & 5 & 6 & 4 & 1 & 1 & 1 \\
\hline \multirow{5}{*}{$\begin{array}{l}f_{9} \\
(D=30)\end{array}$} & Mean & 3.257708808 & 5.701336524 & 0.000124894 & 0 & $3.10916 E-59$ & 0 \\
\hline & Worst & 1.752511346 & 2.663533038 & $4.35934 E-05$ & 0 & $1.26282 E-58$ & $\mathbf{0}$ \\
\hline & Best & 7.423856504 & 13.2677015 & 0.000220385 & 0 & $6.7331 E-58$ & 0 \\
\hline & Worst & 0.503649602 & 1.083438608 & $5.01768 E-05$ & 0 & $4.23177 E-67$ & $\mathbf{0}$ \\
\hline & Rank & 5 & 6 & 4 & 1 & 3 & 1 \\
\hline
\end{tabular}


TABLE 4: Continued.

\begin{tabular}{|c|c|c|c|c|c|c|c|}
\hline \multirow{2}{*}{$\begin{array}{l}\text { Test } \\
\text { functions }\end{array}$} & \multirow{2}{*}{ Result } & \multicolumn{6}{|c|}{ Algorithm } \\
\hline & & PSO & BA & $\mathrm{DE}$ & WDO & GAPSO & WDO-DE \\
\hline \multirow{5}{*}{$\begin{array}{l}f_{10} \\
(D=2)\end{array}$} & Mean & 0.397887358 & 0.397887361 & 0.397887358 & 0.507404422 & 0.411708093 & 0.397887358 \\
\hline & Std. & $3.36448 E-16$ & $4.03531 E-09$ & $3.36448 E-16$ & 0.134086235 & 0.008420321 & $3.36448 E-16$ \\
\hline & Worst & 0.397887358 & 0.397887373 & 0.397887358 & 1.01191027 & 0.427364689 & 0.397887358 \\
\hline & Best & 0.397887358 & 0.397887358 & 0.397887358 & 0.407529279 & 0.398014086 & 0.397887358 \\
\hline & Rank & 1 & 4 & 1 & 6 & 5 & 1 \\
\hline \multirow{5}{*}{$\begin{array}{l}f_{11} \\
(D=2)\end{array}$} & Mean & -1 & -0.04000324 & -1 & -0.96070704 & -0.99999566 & -1 \\
\hline & Std. & 0 & 0.197947995 & 0 & 0.039519354 & $7.81918 E-06$ & 0 \\
\hline & Worst & -1 & 0 & -1 & -0.82029884 & -0.99995866 & -1 \\
\hline & Best & -1 & -1 & -1 & -0.99846688 & -1 & -1 \\
\hline & Rank & 1 & 6 & 1 & 5 & 4 & 1 \\
\hline \multirow{5}{*}{$\begin{array}{l}f_{12} \\
(D=2)\end{array}$} & Mean & 3 & 7.320000769 & 3 & 7.838633652 & 3 & 3 \\
\hline & Std. & $4.29203 E-15$ & 9.998857372 & $3.99781 E-15$ & 4.897011481 & $4.12124 E-15$ & $3.48119 \mathrm{E}-15$ \\
\hline & Worst & 3 & 30.00000257 & 3 & 19.47699347 & 3 & 3 \\
\hline & Best & 3 & 3.000000005 & 3 & 3.024309606 & 3 & 3 \\
\hline & Rank & 4 & 5 & 2 & 6 & 3 & 1 \\
\hline \multirow{5}{*}{$\begin{array}{l}f_{13} \\
(D=2)\end{array}$} & Mean & -1.03162845 & -0.95001198 & -1.03162845 & -1.02310481 & -1.03162845 & -1.03162845 \\
\hline & Std. & $2.24299 E-16$ & 0.247335237 & $2.24299 E-16$ & 0.010122307 & $2.24299 E-16$ & $3.58878 E-16$ \\
\hline & Worst & -1.03162845 & -0.21546376 & -1.03162845 & -0.97714288 & -1.03162845 & -1.03162845 \\
\hline & Best & -1.03162845 & -1.03162845 & -1.03162845 & -1.03147363 & -1.03162845 & -1.03162845 \\
\hline & Rank & 1 & 6 & 1 & 5 & 1 & 4 \\
\hline \multirow{5}{*}{$\begin{array}{l}f_{14} \\
(D=3)\end{array}$} & Mean & -3.86199199 & -3.86277843 & -3.86277979 & -3.73158405 & -3.86277979 & -3.86277979 \\
\hline & Std. & 0.002387392 & $8.83669 E-07$ & $4.93458 E-15$ & 0.076497205 & $4.93458 E-15$ & $4.18232 E-15$ \\
\hline & Worst & -3.8549018 & -3.86277528 & -3.86277979 & -3.52239202 & -3.86277979 & -3.86277979 \\
\hline & Best & -3.86277979 & -3.86277961 & -3.86277979 & -3.85565215 & -3.86277979 & -3.86277979 \\
\hline & Rank & 5 & 4 & 2 & 6 & 2 & 1 \\
\hline \multirow{5}{*}{$\begin{array}{l}f_{15} \\
(D=10)\end{array}$} & Mean & -7.67025031 & -5.6907234 & -9.66015172 & -5.16498045 & -6.53572784 & -9.56777535 \\
\hline & Std. & 0.781635349 & 1.010252541 & $4.39534 E-16$ & 0.511107324 & 0.820311453 & 0.060299252 \\
\hline & Worst & -5.60672009 & -3.1283389 & -9.66015172 & -3.93161423 & -4.57905773 & -9.32718849 \\
\hline & Best & -9.0444038 & -8.11751567 & -9.66015172 & -6.35730534 & -8.47351627 & -9.64648266 \\
\hline & Rank & 3 & 5 & 1 & 6 & 4 & 2 \\
\hline Average rank & & 4.06 & 5.06 & 3 & 3.33 & 2.8 & 1.33 \\
\hline Overall rank & & 5 & 6 & 3 & 4 & 2 & 1 \\
\hline
\end{tabular}

results, the optimal fitness value, the worst fitness value, and rank results between the algorithms of 50 independent runs for $f_{1} \sim f_{15}$ are shown in Table 4 . Bold and italicized results mean that WDO-DE is better. Population size of other algorithms is 100. Max number of iterations of all tests is 1000 .

For the low-dimensional case, according to Table 4, test results of WDO-DE are better than the other algorithms except $f_{5}, f_{13}$, and $f_{15}$. For $f_{5}$ and $f_{15}$, the $\mathrm{DE}$ algorithm gives the better results. Although the result of $f_{13}$ function is worse than GA-PSO, DE, and PSO algorithm, it has already reached the theoretical optimal value. What is more, WDO-DE can find the theoretical optimum values for twelve benchmark functions $\left(f_{1} \sim f_{4}, f_{7} \sim f_{14}\right)$ and has a very strong robustness. The novel hybrid global optimization algorithm is better than the original algorithm.

In the last, we calculated the average rank based on these fifteen functions' ranking [18]. Then, we rank the average rank and obtain the overall rank. From the average rank of each algorithm, we can learn that WDO-DE is very robust and efficient.

For the benchmark function $f_{11}$, the solution of WDO$\mathrm{DE}$ algorithm is the closest to the theoretical optimal solution; for the benchmark function $f_{12} \sim f_{14}$, the original WDO algorithm cannot be close to the theoretical optimal solution; however the WDO-DE algorithm is close to the theoretical optimal solution (Table 5). And we can find that the convergence speed of the WDO-DE algorithm is quicker than other algorithms. In benchmark functions $f_{11}$ and $f_{13}$, the WDO$\mathrm{DE}$ algorithm has converged within 100 generations. And in benchmark functions $f_{12}$ and $f_{14}$, the WDO-DE algorithm also has converged within 200 generations.

Meanwhile, Figures 1-15 have shown the evolutionary process of fitness value (the vertical axis is logarithmic fitness value). And Figures 16-30 are the ANOVA tests of the global 


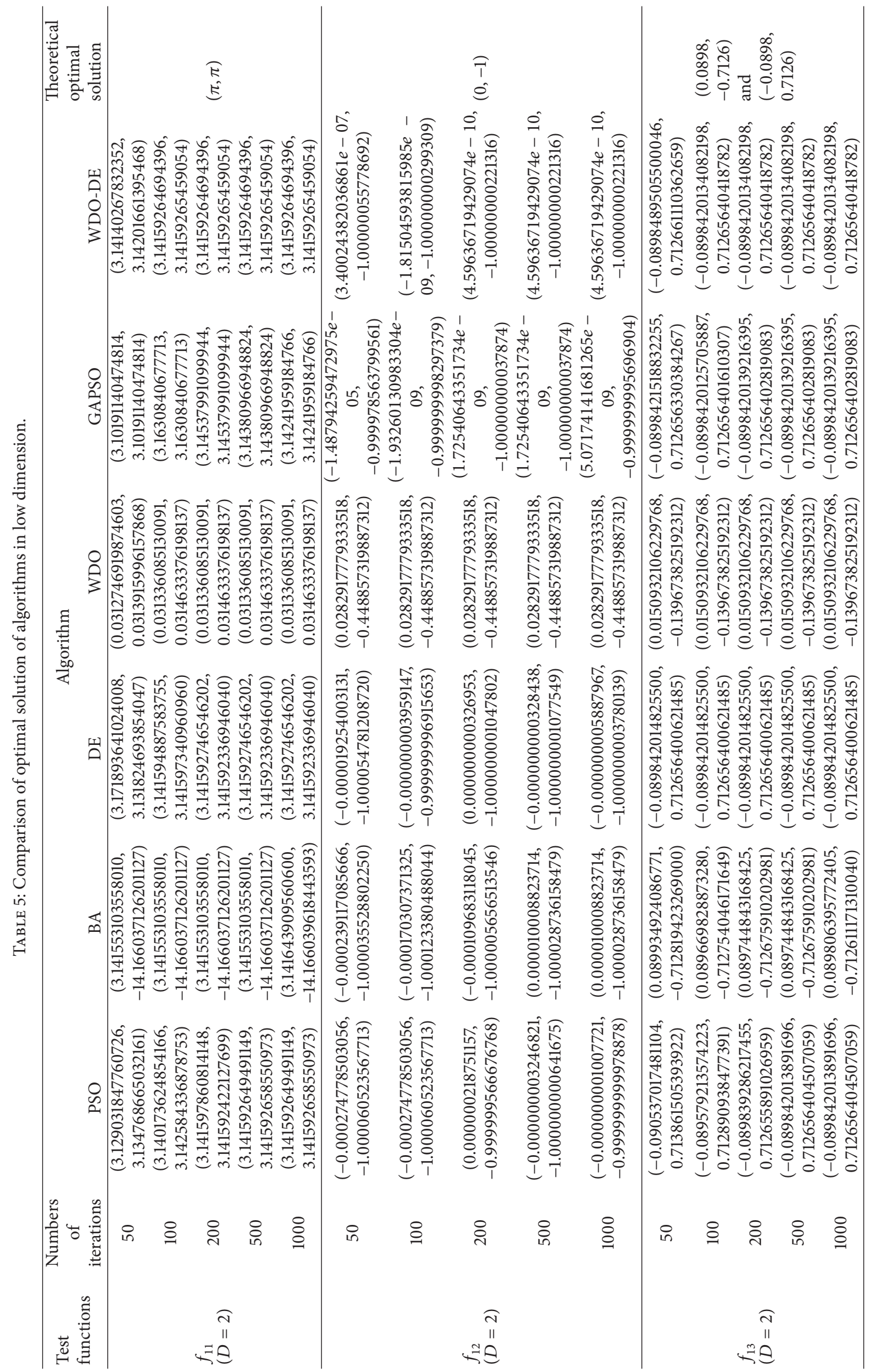




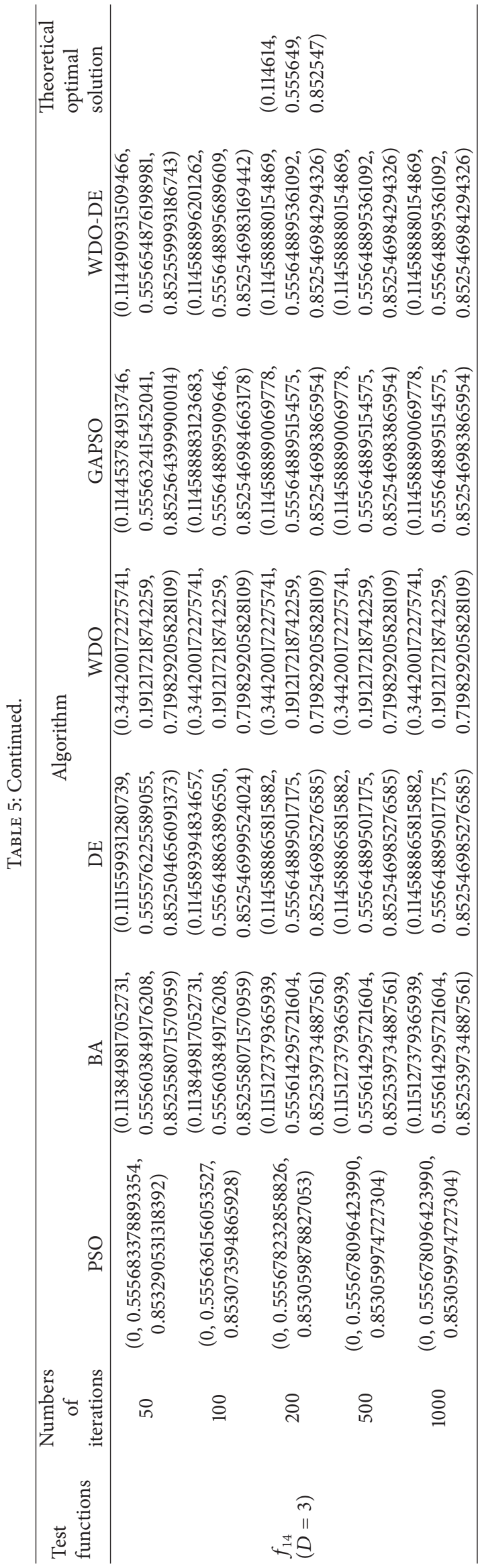




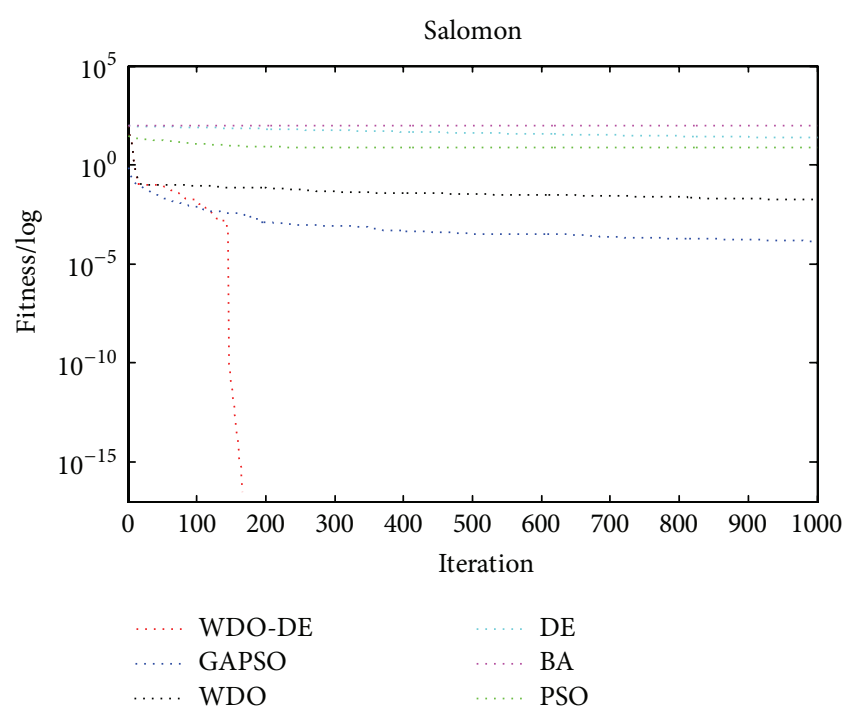

FIGURE 34: Comparison of performance of algorithms for minimization of $f_{4}(D=300)$.

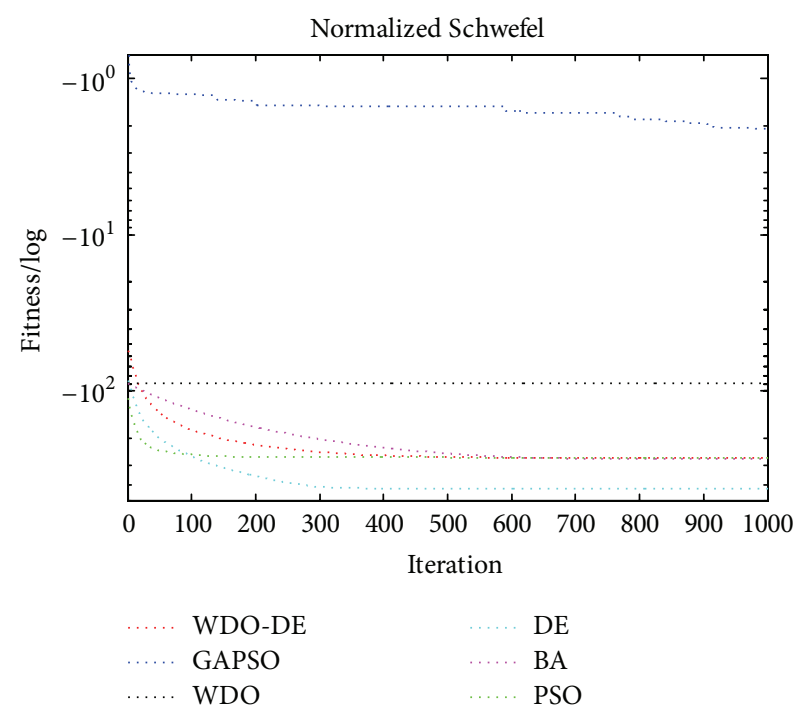

FIGURE 35: Comparison of performance of algorithms for minimization of $f_{5}(D=100)$.

minimum. As can be seen from Figures 1-15, WDO-DE algorithm can converge within the maximum number of iterations except $f_{5}$ and $f_{15}$, and it has a faster global convergence speed in many functions and higher convergence precision. From the evolutionary process of fitness value it can be seen that the WDO-DE algorithm has a strong ability to find the optimal solutions. Moreover, as seen from Figures 1630 , we can learn that WDO-DE is the most robust in these algorithms. Therefore, WDO-DE is an effective and feasible solution for optimization problems in low-dimensional case.

In order to test the optimization ability of the algorithms in high-dimensional space, this paper selects several different dimensions for tests [15]. Among them, $f_{3}$ and $f_{5}$ were set to 100 dimensions, $f_{4}$ and $f_{7}$ set to 300 dimensions, $f_{1}$ and $f_{8}$ set

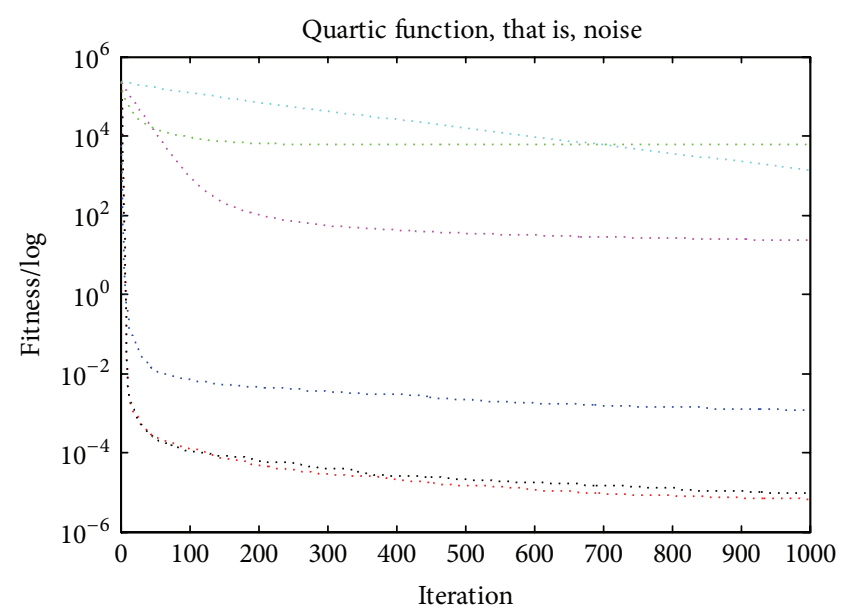

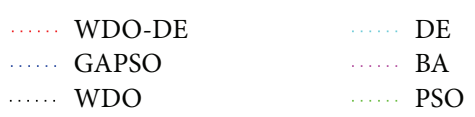

FIGURE 36: Comparison of performance of algorithms for minimization of $f_{6}(D=1000)$.

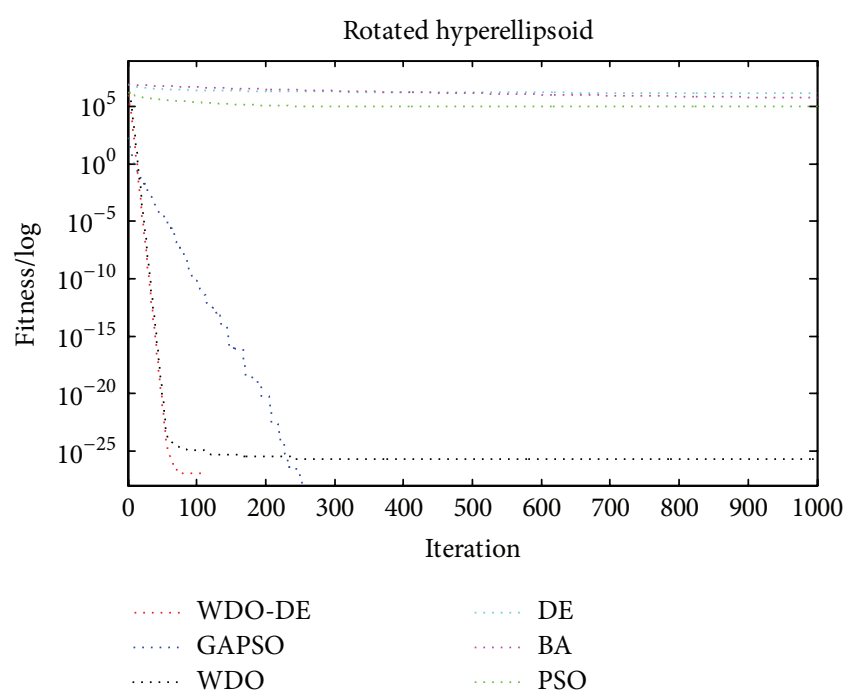

FIGURE 37: Comparison of performance of algorithms for minimization of $f_{7}(D=300)$.

to 500 dimensions, and $f_{2}, f_{6}$, and $f_{9}$ set to 1000 dimensions. In all the tests, the max number of iterations is 1000, and the set of other parameters is the same. The mean results, standard deviation (Std.) results, the optimal fitness value, the worst fitness value, and rank results between the algorithms of 50 independent runs for $f_{1} \sim f_{9}$ are shown in Table 6 .

For the high-dimensional case, as seen from Table 6, test results of WDO-DE are better than the other algorithms except $f_{5}$. For $f_{5}$, the results of $f_{5}$ function are secondary to $\mathrm{DE}$ algorithm; although the $\mathrm{DE}$ algorithm gives better results, it has not reached the theoretical optimal value. WDO-DE can find the theoretical optimum values for seven benchmark functions $\left(f_{1} \sim f_{4}, f_{7} \sim f_{9}\right)$ and has a very strong robustness. This indicates that WDO-DE is very robust and efficient. 
TABLE 6: Comparison of performance of algorithms in high dimension.

\begin{tabular}{|c|c|c|c|c|c|c|c|}
\hline \multirow{2}{*}{$\begin{array}{l}\text { Test } \\
\text { functions }\end{array}$} & \multirow{2}{*}{ Result } & \multicolumn{6}{|c|}{ Algorithm } \\
\hline & & PSO & BA & $\mathrm{DE}$ & WDO & GAPSO & WDO-DE \\
\hline \multirow{5}{*}{$\begin{array}{l}f_{1} \\
(D=500)\end{array}$} & Mean & 5942.812635 & 690529.75 & 7833.289879 & 0 & $3.8474 E-114$ & 0 \\
\hline & Std. & 2010.942412 & 16105.47575 & 307.7206962 & 0 & $2.6148 E-113$ & 0 \\
\hline & Worst & 12148.04486 & 721605.363 & 8350.202931 & 0 & $1.8493 E-112$ & 0 \\
\hline & Best & 1477.889332 & 637825.2912 & 7208.53767 & 0 & $5.6298 E-132$ & 0 \\
\hline & Rank & 4 & 6 & 5 & 1 & 3 & 1 \\
\hline \multirow{5}{*}{$\begin{array}{l}f_{2} \\
(D=1000)\end{array}$} & Mean & 6.021999854 & 19.58454162 & 6.556491165 & $8.88178 E-16$ & $8.88178 E-16$ & $8.88178 \mathrm{E}-16$ \\
\hline & Std. & 0.963645094 & 0.072035802 & 0.086504851 & 0 & 0 & o \\
\hline & Worst & 7.69115243 & 20.05614049 & 6.718429507 & $8.88178 E-16$ & $8.88178 E-16$ & $8.88178 E-16$ \\
\hline & Best & 3.224297469 & 19.5300771 & 6.318110244 & $8.88178 E-16$ & $8.88178 E-16$ & $8.88178 E-16$ \\
\hline & Rank & 4 & 6 & 5 & 1 & 1 & 1 \\
\hline \multirow{5}{*}{$\begin{array}{l}f_{3} \\
(D=100)\end{array}$} & Mean & 5.151497492 & 1774.455594 & 0.038171893 & 0 & 0 & 0 \\
\hline & Std. & 2.161430833 & 103.0300286 & 0.006252419 & 0 & 0 & $\mathbf{0}$ \\
\hline & Worst & 13.35711745 & 1927.77745 & 0.051473105 & 0 & 0 & 0 \\
\hline & Best & 1.782030264 & 1418.804786 & 0.025514473 & 0 & 0 & 0 \\
\hline & Rank & 5 & 6 & 4 & 1 & 1 & 1 \\
\hline \multirow{5}{*}{$\begin{array}{l}f_{4} \\
(D=300)\end{array}$} & Mean & 7.811873346 & 93.93587335 & 23.2983014 & 0.01799082 & 0.000134214 & 0 \\
\hline & Std. & 1.358065852 & 1.176428355 & 0.51684138 & 0.038789028 & 0.00022591 & $\mathbf{0}$ \\
\hline & Worst & 12.59987335 & 96.09987335 & 24.38078634 & 0.100231302 & 0.001189194 & $\mathbf{0}$ \\
\hline & Best & 5.099873346 & 90.29987335 & 22.11997471 & 0 & $3.67969 E-07$ & $\mathbf{0}$ \\
\hline & Rank & 4 & 6 & 5 & 3 & 2 & 1 \\
\hline \multirow{5}{*}{$\begin{array}{l}f_{5} \\
(D=100)\end{array}$} & Mean & -188.353807 & -225.492975 & -415.031229 & -49.9853541 & -2.07455021 & -266.617912 \\
\hline & Std. & 24.35490997 & 13.25383666 & 1.616785061 & 9.085364316 & 1.865239717 & 16.69768835 \\
\hline & Worst & -110.804763 & -196.772214 & -409.751888 & -29.977684 & -0.9468723 & -229.62838 \\
\hline & Best & -231.936138 & -260.621897 & -417.256124 & -71.336055 & -8.7435685 & -294.826296 \\
\hline & Rank & 4 & 3 & 1 & 5 & 6 & 2 \\
\hline \multirow{5}{*}{$\begin{array}{l}f_{6} \\
(D=1000)\end{array}$} & Mean & 6096.609068 & 23.0239015 & 1384.433152 & $9.50848 E-06$ & 0.001216642 & $6.70813 E-06$ \\
\hline & Std. & 3404.371174 & 1.515569145 & 74.20351119 & $1.03931 E-05$ & 0.000874723 & $7.15954 \mathrm{E}-06$ \\
\hline & Worst & 14502.3679 & 27.87497256 & 1594.24402 & $4.67328 E-05$ & 0.003668518 & $3.54361 \mathrm{E}-05$ \\
\hline & Best & 457.2497659 & 19.88875782 & 1219.60675 & $1.46012 E-07$ & $1.20405 E-05$ & $3.50446 \mathrm{E}-07$ \\
\hline & Rank & 6 & 4 & 5 & 2 & 3 & 1 \\
\hline \multirow{5}{*}{$\begin{array}{l}f_{7} \\
(D=300)\end{array}$} & Mean & 97529.8314 & 570894.7164 & 1506962.5 & $1.94072 E-26$ & $1.1197 E-114$ & 0 \\
\hline & Std. & 49025.64187 & 99081.42372 & 90799.2443 & $1.3723 E-25$ & $6.0113 E-114$ & 0 \\
\hline & Worst & 254179.9951 & 781652.5658 & 1740971.299 & $9.70362 E-25$ & $3.9713 E-113$ & 0 \\
\hline & Best & 39232.88764 & 383943.7522 & 1315295.793 & 0 & $2.0359 E-133$ & 0 \\
\hline & Rank & 4 & 5 & 6 & 3 & 2 & 1 \\
\hline \multirow{5}{*}{$\begin{array}{l}f_{8} \\
(D=500)\end{array}$} & Mean & 3250.396382 & 4161.811265 & 3678.744509 & 0 & 0 & 0 \\
\hline & Std. & 478.6226817 & 131.4154437 & 45.71686166 & 0 & 0 & 0 \\
\hline & Worst & 4235.766671 & 4449.362401 & 3755.802488 & 0 & 0 & 0 \\
\hline & Best & 2253.591512 & 3822.595502 & 3563.581287 & 0 & 0 & 0 \\
\hline & Rank & 4 & 6 & 5 & 1 & 1 & 1 \\
\hline \multirow{5}{*}{$\begin{array}{l}f_{9} \\
(D=1000)\end{array}$} & Mean & 201.0787949 & 703.6914404 & 751.9162928 & 0 & $2.40675 E-58$ & 0 \\
\hline & Worst & 51.1605331 & 35.62374915 & 15.21931169 & 0 & $8.18033 E-58$ & $\mathbf{0}$ \\
\hline & Best & 385.1517001 & 765.0787636 & 779.6415024 & 0 & $4.24827 E-57$ & 0 \\
\hline & Worst & 87.92253474 & 626.8641799 & 720.1039204 & 0 & $1.30498 E-65$ & 0 \\
\hline & Rank & 4 & 5 & 6 & 1 & 3 & 1 \\
\hline Average rank & & 4.33 & 5.22 & 4.67 & 2 & 2.44 & 1.11 \\
\hline Overall rank & & 4 & 6 & 5 & 2 & 3 & 1 \\
\hline
\end{tabular}




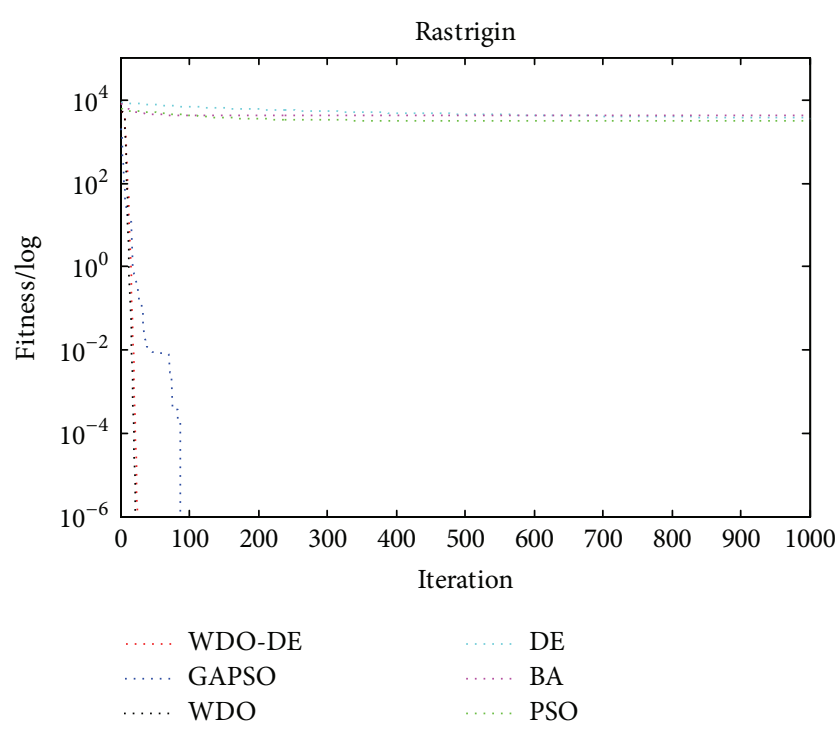

FIGURE 38: Comparison of performance of algorithms for minimization of $f_{8}(D=500)$.

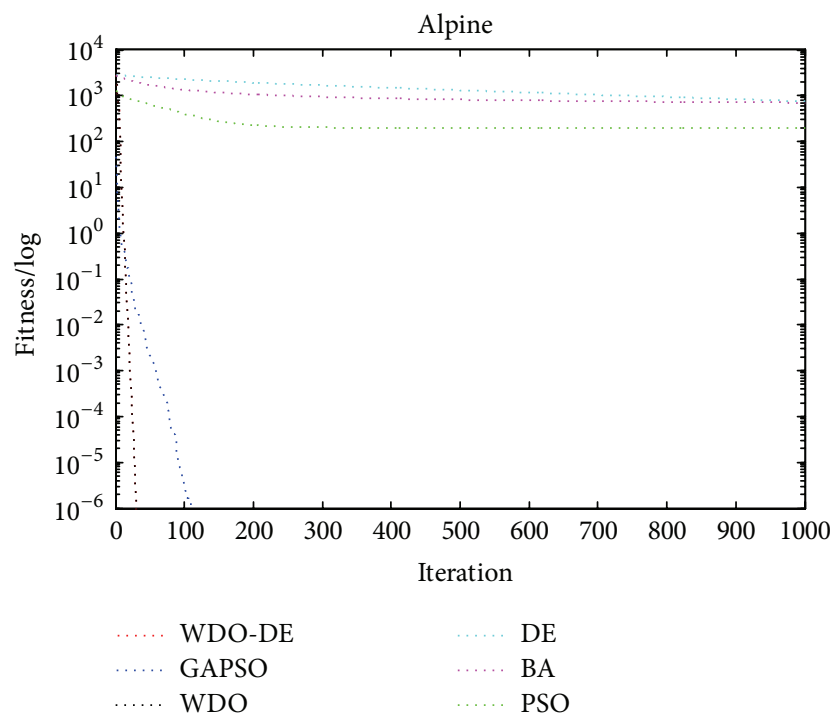

Figure 39: Comparison of performance of algorithms for minimization of $f_{9}(D=1000)$.

The same as before, we calculated the average rank based on these nine functions' ranking. Then, we rank the average rank and obtain the overall rank. From the average rank of each algorithm, we can learn that WDO-DE is very robust and efficient.

The same as before, Figures 31-39 have shown the evolutionary process of fitness value (the vertical axis is logarithmic fitness value). And Figures 40-48 are the ANOVA tests of the global minimum. As can be seen from Figures 31-39, WDO$\mathrm{DE}$ algorithm has the fastest global convergence speed and the highest convergence precision in all of these functions only except $f_{5}$. From the evolutionary process of fitness value it can be seen that the WDO-DE algorithm has a strong

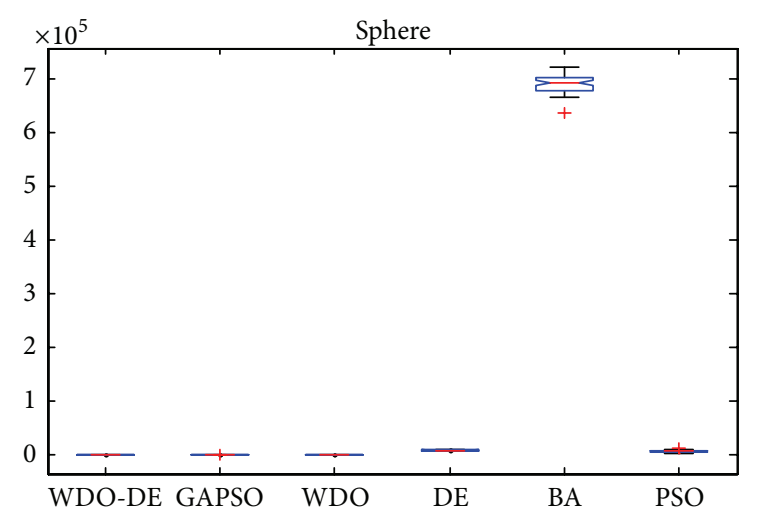

FIGURE 40: ANOVA tests of the global minimum values for $f_{1}(D=$ $500)$.

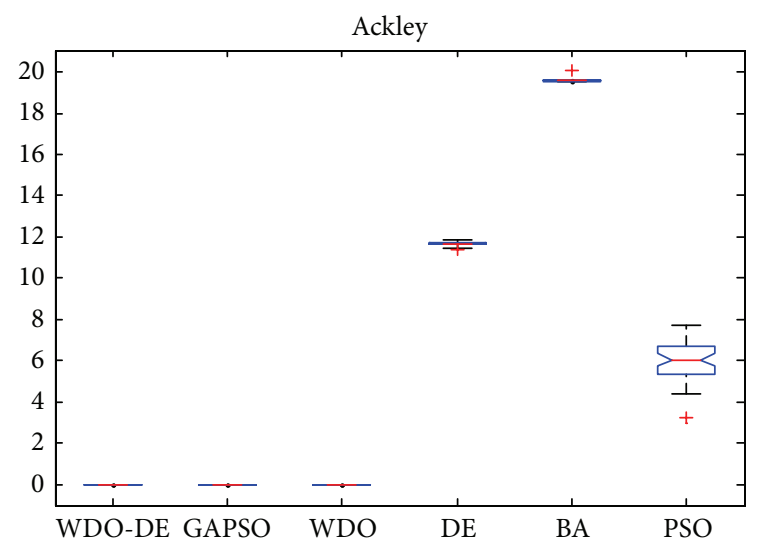

FIgURE 41: ANOVA tests of the global minimum values for $f_{2}(D=$ 1000).

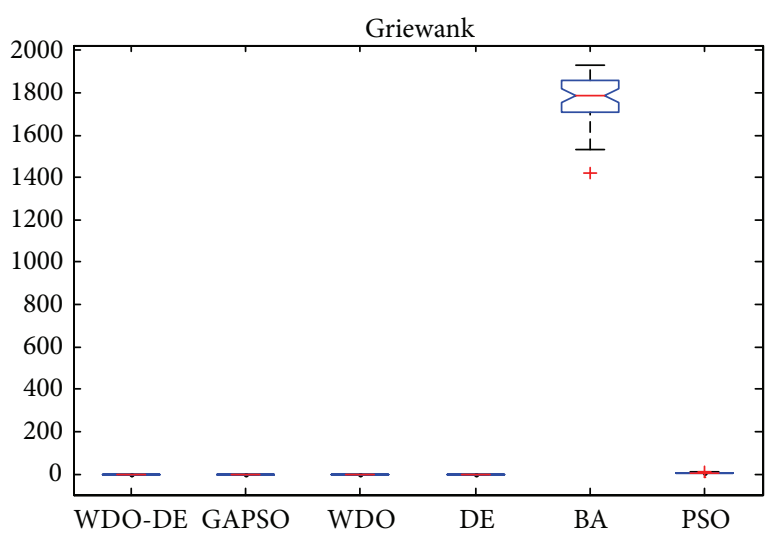

FIGURE 42: ANOVA tests of the global minimum values for $f_{3}(D=$ 100).

ability to find the optimal solutions. Moreover, as seen from Figures $40-48$, we can learn that WDO-DE is the most robust in these algorithms. Therefore, WDO-DE is also an effective and feasible solution for optimization problems in high-dimensional case. 


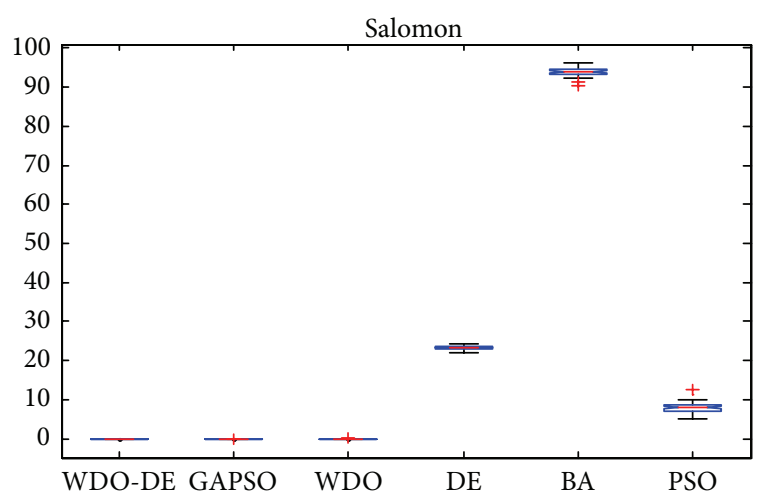

Figure 43: ANOVA tests of the global minimum values for $f_{4}(D=$ 300).

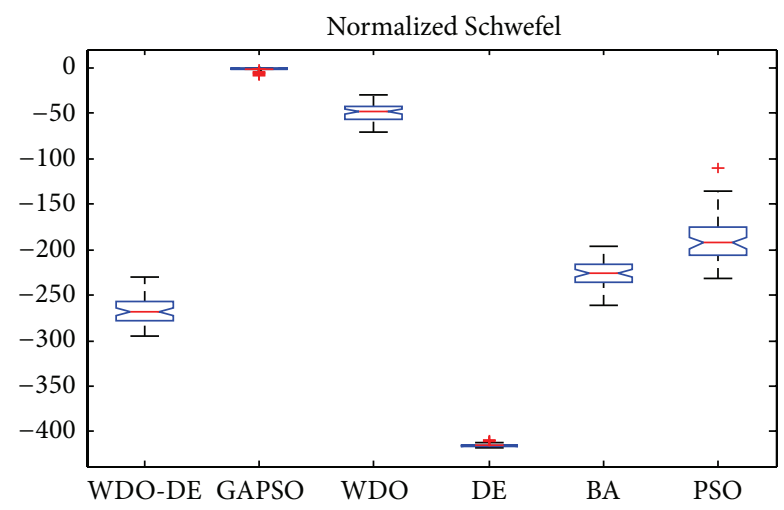

FIGURE 44: ANOVA tests of the global minimum values for $f_{5}(D=$ 100).

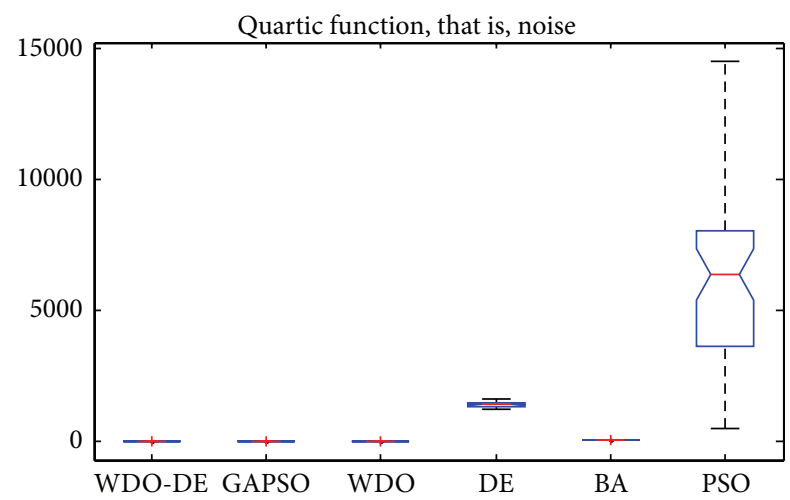

FIGURE 45: ANOVA tests of the global minimum values for $f_{6}(D=$ 1000).

\section{Conclusion and Future Research}

In this paper, we present a new hybrid global optimization algorithm called WDO-DE, which is based on the wind driven optimization (WDO) and differential evolution (DE) for solving optimization problems. We use 15 benchmark functions which contain unimodal, multimodal, low-dimensional, and high-dimensional unconstrained test functions to test the performance of WDO-DE algorithm.

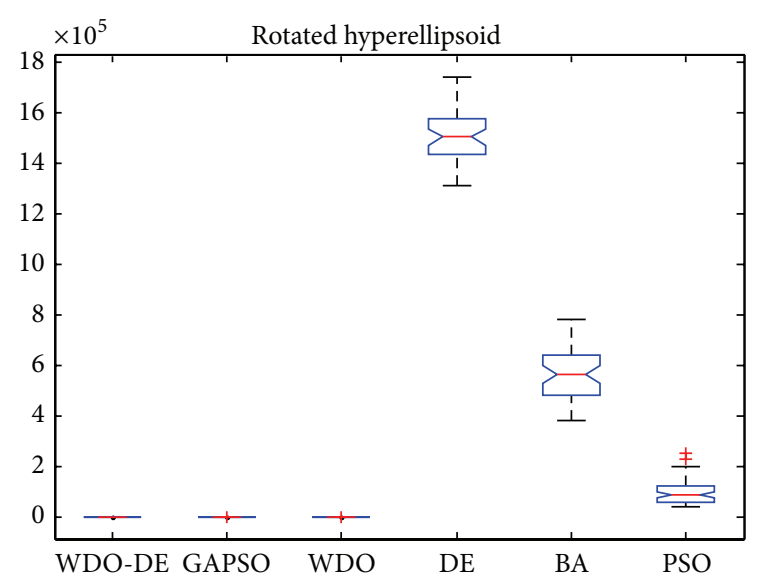

FIgURE 46: ANOVA tests of the global minimum values for $f_{7}(D=$ $300)$.

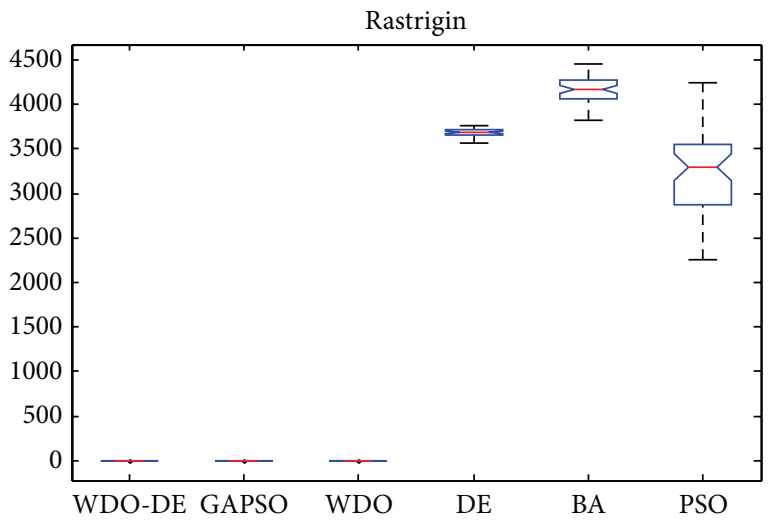

FIGURE 47: ANOVA tests of the global minimum values for $f_{8}(D=$ 500).

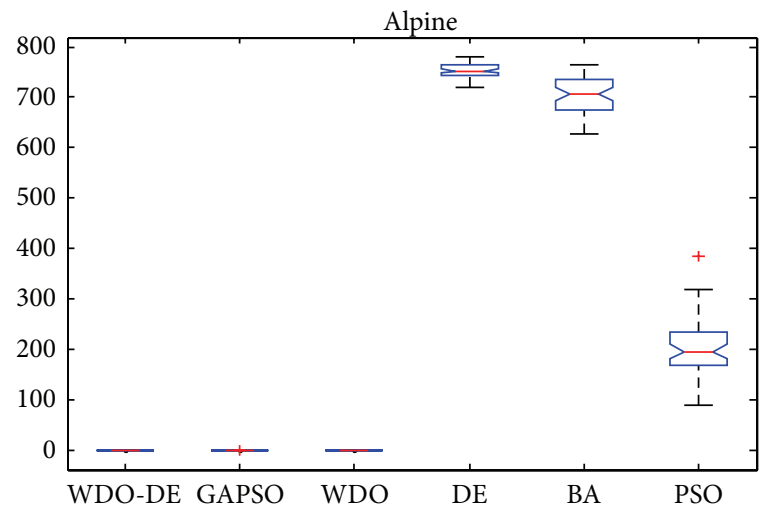

Figure 48: ANOVA tests of the global minimum values for $f_{9}(D=$ 1000).

The WDO-DE algorithm can converge within the maximum number of iterations in most functions. In comparison with the GA-PSO, WDO, DE, BA, and PSO, the WDO-DE algorithm is more effective in finding better solutions and the convergence speed and precision of WDO-DE are higher. It is an effective and reliable global optimization algorithm. 
Although in this paper the hybrid WDO-DE algorithm was implemented only for function optimization, in the field of optimization, there are still many aspects worthy of our study. Firstly, the hybrid algorithm proposed in this paper is based on the continuous space optimization. The future research may concentrate on discrete WDO algorithm. We can utilize many discretized strategies to discretize WDO algorithm to solve a problem characterized by discretevalued design variables. Secondly, in engineering application, production management, and national defense construction, many optimization problems are multiobjective optimization problems, which are widely used in practical engineering. We would apply our proposed hybrid approach to solve multiobjective optimization problem. Lastly, we will learn more algorithms which have better optimization performance and analyze their characteristics. We would develop new hybrid approaches to solve the optimization problems raised above.

\section{Conflict of Interests}

The authors declare that there is no conflict of interests regarding the publication of this paper.

\section{Acknowledgments}

This work is supported by National Science Foundation of China under Grants nos. 61165015 and 61463007 and the Innovation Project of Guangxi Graduate Education.

\section{References}

[1] D. E. Golberg, Genetic Algorithms in Search, Optimization, and Machine Learning, Addion Wesley, 1989.

[2] R. C. Eberhart and J. A. Kennedy, "A new optimizer using particle swarm theory," in Proceedings of the 6th International Symposium on Micro Machine and Human Science (MHS '95), pp. 39-43, IEEE, Nagoya, Japan, October 1995.

[3] J. Kennedy, "Particle swarm optimization," in Encyclopedia of Machine Learning, pp. 760-766, Springer, New York, NY, USA, 2010.

[4] M. Dorigo, V. Maniezzo, and A. Colorni, "Ant system: optimization by a colony of cooperating agents," IEEE Transactions on Systems, Man, and Cybernetics, Part B: Cybernetics, vol. 26, no. 1, pp. 29-41, 1996.

[5] R. Storn and K. Price, "Differential evolution-a simple and efficient heuristic for global optimization over continuous spaces," Journal of Global Optimization, vol. 11, no. 4, pp. 341359, 1997.

[6] X. S. Yang, "Firefly algorithms for multimodal optimization," in Stochastic Algorithms: Foundations and Applications, pp. 169178, Springer, Berlin, Germany, 2009.

[7] X. S. Yang, "A new metaheuristic bat-inspired algorithm," in Nature Inspired Cooperative Strategies for Optimization (NICSO 2010), pp. 65-74, Springer, Berlin, Germany, 2010.

[8] X.-S. Yang and S. Deb, "Cuckoo search via Lévy flights," in Proceedings of the World Congress on Nature \& Biologically Inspired Computing (NaBIC '09), pp. 210-214, IEEE, Coimbatore, India, December 2009.
[9] X. S. Yang, "Flower pollination algorithm for global optimization," in Unconventional Computation and Natural Computation, pp. 240-249, Springer, Berlin, Germany, 2012.

[10] Z. Bayraktar, M. Komurcu, and D. H. Werner, "Wind Driven Optimization (WDO): a novel nature-inspired optimization algorithm and its application to electromagnetics," in Proceedings of the Antennas and Propagation Society International Symposium (APSURSI), pp. 1-4, IEEE, Toronto, Canada, July 2010.

[11] C.-F. Juang, "A hybrid of genetic algorithm and particle swarm optimization for recurrent network design," IEEE Transactions on Systems, Man, and Cybernetics, Part B: Cybernetics, vol. 34, no. 2, pp. 997-1006, 2004.

[12] G. Wang, L. Guo, H. Duan, H. Wang, L. Liu, and M. Shao, "A hybrid metaheuristic DE/CS algorithm for UCAV threedimension path planning," The Scientific World Journal, vol. 2012, Article ID 583973, 11 pages, 2012.

[13] N. Holden and A. A. Freitas, "A hybrid particle swarm/ant colony algorithm for the classification of hierarchical biological data," in Proceedings of the IEEE Swarm Intelligence Symposium (SIS '05), pp. 100-107, June 2005.

[14] H. Riehl, Introduction to the Atmosphere, McGraw-Hill, 1978.

[15] C. D. Ahrens, Meteorology Today: An Introduction to Weather, Climate, and the Environment, Thomson-Brooks/Cole, Belmont, Calif, USA, 7th edition, 2003.

[16] H. Xia, Z. Wang, and Y. Zhou, "Double-population differential evolution based on logistic model," Journal of Information and Computational Science, vol. 11, no. 15, pp. 5549-5557, 2014.

[17] L. Li, Y. Zhou, and J. Xie, "A free search krill herd algorithm for functions optimization," Mathematical Problems in Engineering, vol. 2014, Article ID 936374, 21 pages, 2014.

[18] X. Li, J. Zhang, and M. Yin, "Animal migration optimization: an optimization algorithm inspired by animal migration behavior," Neural Computing and Applications, vol. 24, no. 7-8, pp. 18671877, 2014.

[19] Z. Bayraktar, M. Komurcu, J. A. Bossard, and D. H. Werner, "The wind driven optimization technique and its application in electromagnetics," IEEE Transactions on Antennas and Propagation, vol. 61, no. 5, pp. 2745-2757, 2013.

[20] A. K. Bhandari, V. K. Singh, A. Kumar, and G. K. Singh, "Cuckoo search algorithm and wind driven optimization based study of satellite image segmentation for multilevel thresholding using Kapur's entropy," Expert Systems with Applications, vol. 41, no. 7, pp. 3538-3560, 2014. 


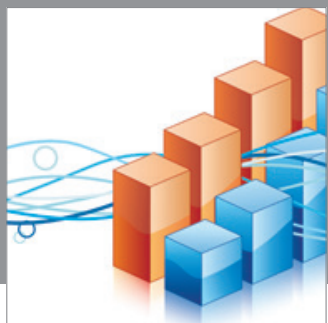

Advances in

Operations Research

mansans

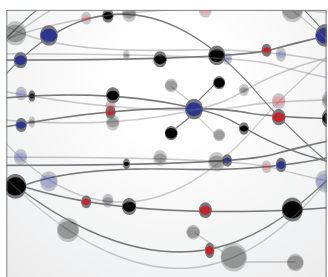

The Scientific World Journal
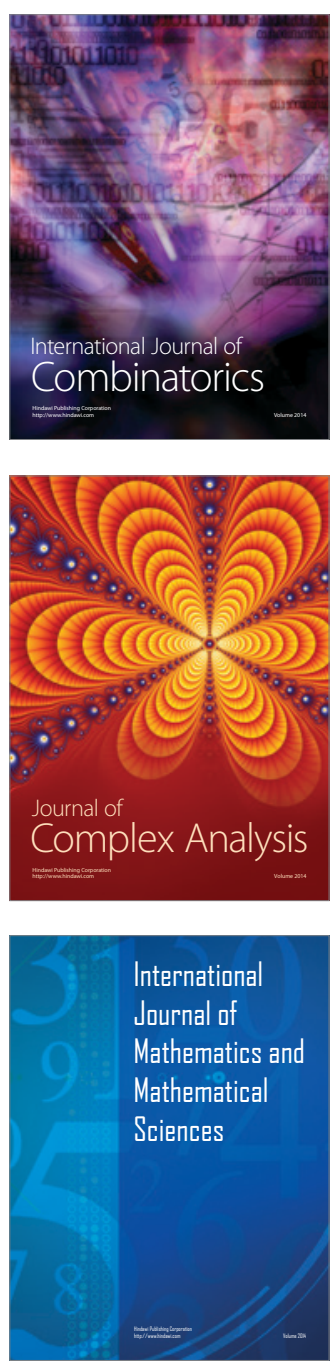
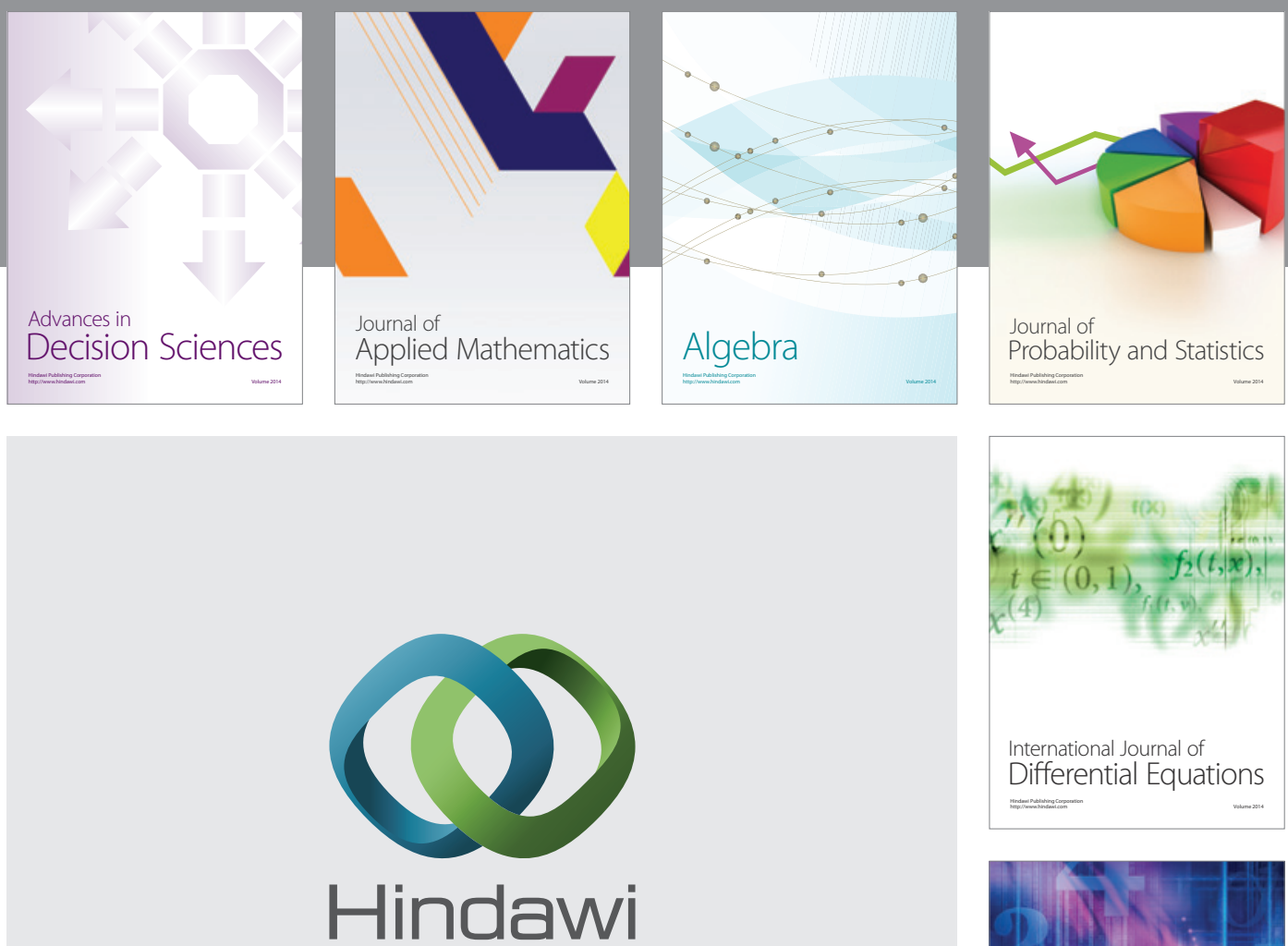

Submit your manuscripts at http://www.hindawi.com
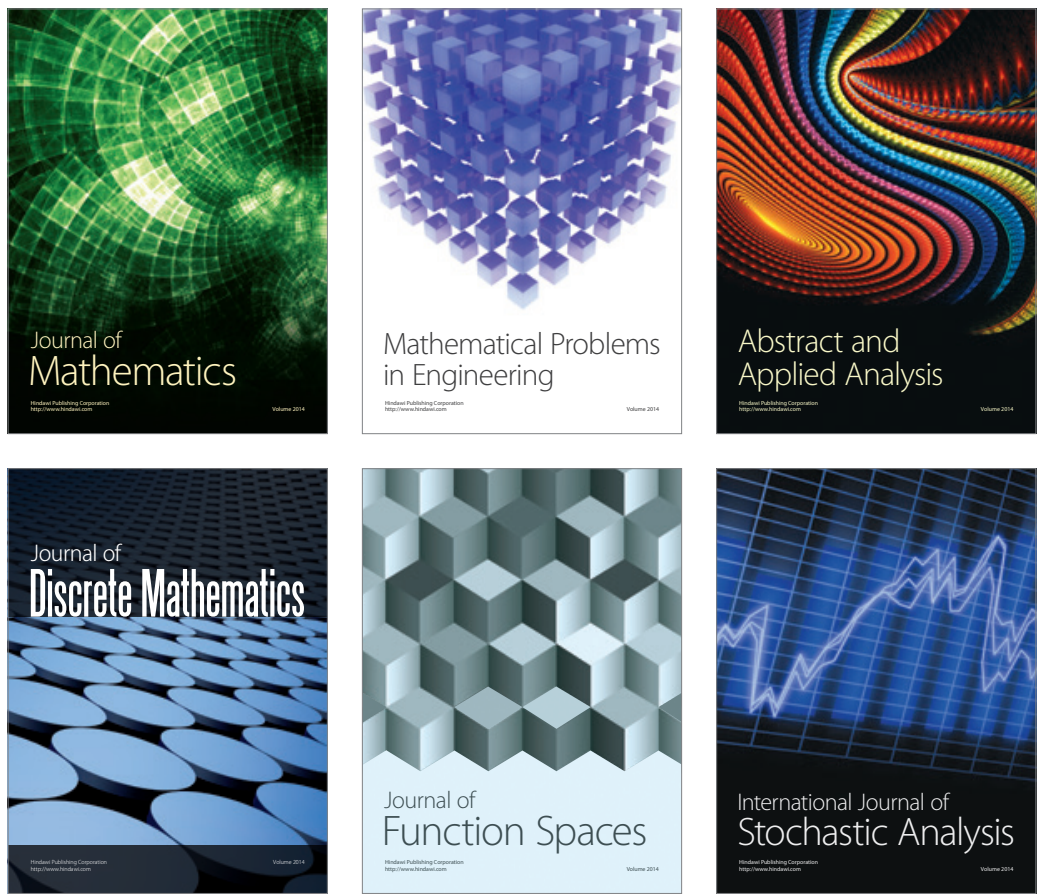

Journal of

Function Spaces

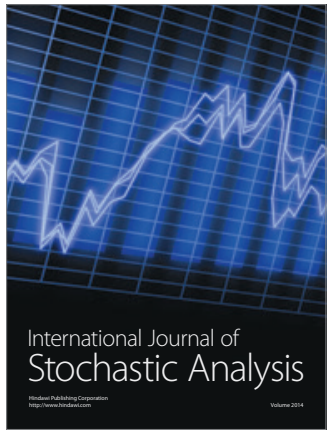

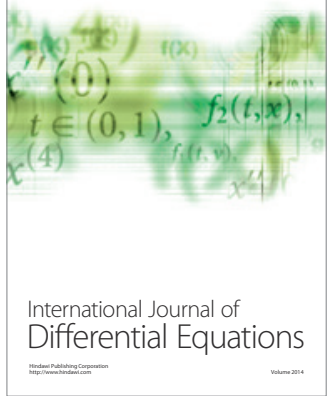
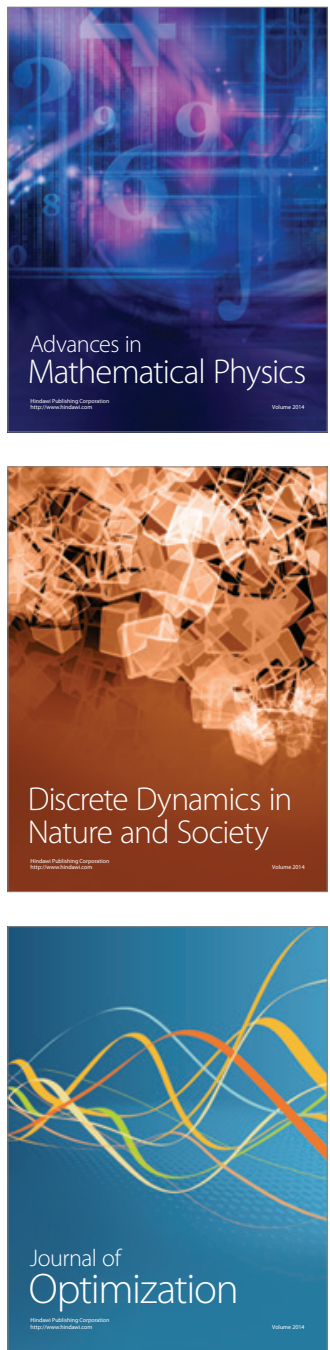